Published in final edited form as:

J Opt Soc Am A Opt Image Sci Vis. 2013 April 1; 30(4): 769-782.

\title{
Quantitative orientation-independent DIC microscope with fast switching shear direction and bias modulation
}

\author{
Michael Shribak \\ Marine Biological Laboratory, 7 MBL St, Woods Hole, MA 02543, USA
}

Michael Shribak: mshribak@mbl.edu

\begin{abstract}
We describe quantitative orientation-independent differential interference contrast (OI-DIC) microscope, which allows the bias retardation to be modulated and shear directions to be switched rapidly without any mechanically moving. The shear direction is switched by regular liquid crystal cell sandwiched between two standard DIC prisms. Another liquid crystal cell modulates the bias. Techniques for measuring parameters of DIC prisms and calibrating the bias are shown. Two sets of raw DIC images with the orthogonal shear directions are captured within a second. Then the quantitative image of optical path gradient distribution within a thin optical section is computed. The gradient data are used to obtain quantitative distribution of optical path, which represents refractive index gradient or height distribution. Computing enhanced regular DIC images with any desired shear direction is also possible.
\end{abstract}

\section{Introduction}

Differential interference contrast (DIC) light microscope is a polarizing beam-shearing interference system in which the interfering beams are sheared by a small amount, generally by somewhat less than the radius of an Airy disk. The DIC technique produces a monochromatic shadow-cast image that displays the gradient of optical paths. Those regions of the specimen where the optical paths increase along a reference direction appear brighter (or darker), while regions where the path differences decrease appear in reverse contrast. As the gradient of optical path grows steeper, image contrast is significantly increased. Another important feature of the DIC technique is that it produces effective optical sectioning. This is particularly obvious when high numerical aperture (NA) objectives are used together with high NA condenser illumination.

The DIC technique was invented by Fransis H. Smith in 1947 [1,2]. He placed between a pair of the crossed polarizers one Wollaston prism at the front focal plane of the condenser and another Wollaston prism in the back focal plane of objective lens. The splitting angles of the prisms $\epsilon_{1}$ and $\epsilon_{2}$ are connected with the focal distances of the condenser and objective $f_{c}$ and $f_{o b}$ and the shear amount $d$ by the following relation:

$$
f_{c} \varepsilon_{1}=f_{o b} \varepsilon_{2}=d
$$

However, in conventional medium- to high-NA objective lenses, the back focal plane is located inside the lens system and therefore not available for insertion of Wollaston prism. The Smith shearing DIC scheme requires special design for the microscope objective lenses so that the Wollaston prism can be incorporated within.

Georges Nomarski, who proposed in 1952 a special polarization prism, took another approach $[3,4]$. He devised prism, which introduces spatial displacement and angular 
deviation of orthogonally polarized beams simultaneously. The Nomarski prism can therefore be placed outside of the objective lens.

The DIC microscopy demonstrates remarkable optical sectioning capability, like confocal microscopy. The contrast in DIC is produced by the optical path difference in a small infocus volume where two interfering beams are spatially separated. Here the beams travel through the different areas of the specimen under investigation. The out-of-focus object introduces practically the same phase disturbance in the both beams because the beams go through almost same area of the specimen. Therefore, the out-of-focus disturbance is suppressed by optical subtraction. It was shown experimentally [5] that the depth of the optical sections, with illumination at $546 \mathrm{~nm}$ and $0.95 \mathrm{NA}$ objective lens, could be $0.25 \mu \mathrm{m}$ for DIC microscopy. Using formulas from a reference [6] one can find that, at the same condition, a theoretical depth of field of conventional brightfield microscope is $0.8 \mu \mathrm{m}$. The optical section depth becomes thinner if the shear amount is smaller, and the objective and condenser numerical apertures are larger. The narrow optical sectioning DIC phenomenon is similar to removing an out-of-focus haze in the structured illumination microscopy (SIM) [6]. The SIM employs a single-spatial-frequency grid pattern, which is projected onto the object under investigation. Raw images are taken at three spatial positions of the grid. The out-of-focus picture of the object does not depend on the pattern position. As a result, the out-of-focus haze in SIM is subtracted computationally.

Application of computation subtraction in the DIC should also improve its sectioning capability even further. The computation subtraction of images with different biases is employed in various techniques, such as polarization modulation DIC (PM-DIC) [7,8], differential detection DIC (D-DIC) with polarizing beamsplitter [9], phase-shifting DIC (PSDIC) [10-12], retardation modulation DIC (RM-DIC) [13-15], and orientation-independent DIC (OI-DIC) [16-18]. In particularly, the PM-DIC removes a background contribution that is insensitive to defocus [8]. It is shown theoretically and confirmed experimentally that a RM-DIC microscope has stronger optical sectioning than a conventional DIC microscope, and the optical section depth is thinner if a Nomarski prism with smaller shear amount is used [15]. Our experiments with the OI-DIC microscope using 100×/1.3NA oil immersion objective lens demonstrated the optical section depth about $0.1 \mu \mathrm{m}$. The corresponding field depth of a bright-field microscope would be $0.5 \mu \mathrm{m}$.

The regular DIC microscope, however, has shortcomings: it requires the proper orientation of a specimen in relation to the optical system in order to achieve best pictures, and its images are not quantitative. In order to obtain quantitative data it is necessary to capture DIC images with orthogonal shear directions. The previous approaches [11, 19-22] used mechanical rotation either of the specimen or DIC prism. This takes considerable time and the obtained images can be misaligned. Therefore the previous methods in the DIC microscopy were not suitable for imaging live cells. To overcome these limitations we have designed and successfully built a new DIC microscope, which allows the bias and shear directions to be switched rapidly without mechanically rotating the specimen or the prisms $[17,18]$. In this system we incorporate algorithms that we have developed. The new OI-DIC technique provides quantitative orientation-independent images of phase (dry mass) and phase gradient with highest fidelity and resolution, which cannot be obtained with any other optical microscope.

\section{Principal optical schematics of orientation-independent differential interference contrast (OI-DIC) microscope}

A regular DIC microscope produces images, which contrast depends on the beam shear direction and phase shift (bias) between the interfering beams. In order to achieve the 
highest contrast of a specimen under investigation the researcher should manually rotate a microscope stage to make the shear direction parallel to the specimen's refractive index gradient, and he/she should optimize the bias by moving a DIC prism laterally or rotating a compensator [6]. The orientation-independent differential interference contrast (OI-DIC) microscope changes the shear direction and the bias quickly and without any mechanical manipulation $[17,18]$. Instead of a single DIC prism the OI-DIC scheme employs two DIC prisms with liquid crystal variable retarder or liquid crystal $90^{\circ}$ polarization rotator in between. This combination creates a beam-shearing assembly, which allows switching the shear direction by $90^{\circ}$ at high speed and without the image misalignment. Another liquid crystal variable retarder works as an optical phase shifter that controls the bias. In principle, instead of liquid crystal cells other means for changing the light polarization can be employed.

Principal optical schematics of OI-DIC microscope for the transmitted and reflected light are shown in Fig. 1 and Fig. 2, correspondently. They include light source with bandpath filter, crossed linear polarizer and analyzer, phase shifter, objective lens and image sensor. The transmitted light schematic contains two beam-shearing assemblies and condenser lens. The reflected setup has one beam-shearing assemblies and semi-transparent beamsplitter. The both schematics could also include a displacement compensator. Orientation of the polarizers and birefringent components are shown in the pictures. The phase shifter can be placed anywhere between polarizers and analyzer. It is necessary to keep in mind that the bias will be doubled if the phase shifter is located between beamsplitter and specimen in the reflected light scheme.

The beam shearing assemblies could employ regular DIC prisms from the microscope. The first prism in assembly is placed in the designed original position and the second DIC prism is displaced along the microscope optical axis by some distance. The large prism displacement causes a strong non-uniformity of intensity distribution across the view filed and reduces the image contrast significantly. One solution of the displacement problem is the using a custom made second DIC prism, which is designed for the specific position. But this way would require knowledge of the DIC prism design and such prism could be very expensive. The second solution is to extract the bare DIC prisms from their original mounts and put them in new holder at close distance. Thickness of each DIC prism is about $1 \mathrm{~mm}$, and thickness of a liquid crystal cell is about $2 \mathrm{~mm}$. A small displacement of the each DIC prism by $2 \sim 3 \mathrm{~mm}$ from the designed axial position would not deteriorate the image quality substantially. The third solution is to use the second DIC prism in its original mount and a special displacement compensator. The compensator corrects misalignment in the interfering beams caused by displacement of the prism. The axial displacement of the DIC prism changes a transversal distance between two beams and does not change angle between them. The compensator introduces the transversal displacement in position of the extraordinary beam that is equal and opposite to the transversal distance change caused by the DIC prism. Such compensating displacement could be created by a thin calcite plane parallel plate with the optic axis inclined at $45^{\circ}$ to the surface, for instance. Detail description of the compensator design is quite cumbersome and it will be done in a future publication.

An example of beam-shearing assembly along with phase shifter is shown in Fig. 3 (center). The picture illustrates the polarization ray tracing in illumination part of the transmitted light microscope (Fig.1). Here we assume that DIC prisms are made of the positive birefringent crystal such as quartz. The diagrams to the left and right in Fig. 3 depict polarization transformations inside the assembly and the corresponding change of shear directions of the output beams. The left column illustrates a case when the beam polarizations between two prisms are preserved and the shear direction is $45^{\circ}$ after the second DIC prism. The right 
column describes a rotation of the output shear direction by $90^{\circ}$ caused by switching the beam polarizations after the first DIC prism.

The schematic consists of linear polarizer $\mathbf{P}$, phase shifter, pair of DIC prisms (Nomarski or Wollaston) DIC1 and DIC2 with orthogonal shear directions, and $90^{\circ}$ polarization rotator. Shear direction of the first prism DIC1 is chosen as the initial X-direction to describe the orientation of the above mentioned optical elements. Azimuth of the transmission axis of the polarizer $\mathbf{P}$ is $45^{\circ}$ in order to create equal intensities of polarization components that become spatially divided by the first prism.

The phase shifter introduces bias $\Gamma$ between the $\mathrm{X}$ - and $\mathrm{Y}$-polarization components. Principal axis of the phase shifter is parallel to the shear direction. Dependence of the phase retardation on the applied voltage has to be smooth enough in order to achieve the required bias change accurately and quickly. The initial bias can be adjusted by sliding of the wedge component of one of the DIC prisms. In principle, the phase shifter can be installed with the same orientation in any place in the assembly. A standard untwisted nematic liquid crystal cell, Freedericksz cell, which works in the Electrically Controlled Birefringence (ECB) mode [23-26], can be employed as phase shifter. The Freedericksz cell configuration is different from the twisted nematic configuration typically used in liquid crystal displays. Conventional Freedericksz ECB cell consists of homogeneously parallel aligned liquid crystal directors. The ECB mode uses the applied voltage to change the tilt of the liquid crystal molecules, as a result, the birefringence is changed as a function of the tilt angle. When no voltage is applied, the liquid crystal molecule's directors are aligned parallel to the cell substrates and the retardance it at a maximum. When a voltage is applied, an electric field is introduced which supplies a torque to the liquid crystal molecules; the retardance is decreased. The molecules near the substrates are not able to fully rotate, and so the retardance does not quite get to zero. Conventional ECB cell has retardance range from 0.1 to two wave $(2 \lambda)$ at $700 \mathrm{~nm}[24,25]$.

It is also possible to use an ECB cell with vertical alignment (VA ECB) as phase shifter $[23,27]$. The liquid crystal molecule's directors are aligned perpendicular (vertical) to the cell substrates if no voltage is applied, and the retardance is at a minimum (zero). Applied voltage turns the liquid crystal directors, and so the retardance is increased. The vertically aligned LC cell exhibits the highest contrast value among all LC cells. Another its merit is fast response speed due to thin cell gap. It does not require a compensation film in order to get zero retardance. The VA ECB has some shortcomings, such as lower retardance range from 0 to half wave $(N / 2)$ at $700 \mathrm{~nm}$, and reduced contrast for off axis rays.

In opposite to the phase shifter, which changes birefringence smoothly in order to obtain the required bias value, the polarization rotator has two states only. In state of OFF the beam passes through the rotator without modifying the $\mathrm{X}$ - and $\mathrm{Y}$ - polarization components. In state of $\mathrm{ON}$ the polarization rotator switches the $\mathrm{X}$ - and $\mathrm{Y}$ - components. The rotator can utilize conventional ECB or VA ECB liquid crystal cell, which is similar to one used by the phase shifter. In this case the principal axis of the LC cell has to be orientated at $45^{\circ}$. The LC cell switches polarization by $90^{\circ}$ (state ON) when the plate retardation is half wave $(N$ 2 ), and preserves beam polarization (state OFF) if the plate retardance is 0 or full wave $(\lambda)$.

It is convenient to utilize a $90^{\circ}$ twisted nematic (TN) cell as the rotator [26,27]. Currently the TN cells are commonly used in liquid crystal displays and shutters. They are not expensive and less sensitive to the wavelength variation then ECB cells. In the TN cells the LC anchoring (or rubbing) direction at the input surface is perpendicular to that of the output surface. In the inactivated state (voltage is below threshold), the local LC director undergoes a continuous twist of $90^{\circ}$ in the region between the plates. Linearly polarized input light 
propagates through and, under ideal circumstances, follows the director helix as it propagates through the LC structure. Lets us assume that LC anchoring direction at the input surface is oriented along the $\mathrm{X}$-axis and anchoring direction at the output surface is oriented along the $\mathrm{Y}$-axis. In this case, light with initial $\mathrm{X}$-polarization emerges from the LC cell with Y-polarization, and light with initial Y-polarization comes out with X-polarization. In order to reduce interaction between $\mathrm{X}$ - and $\mathrm{Y}$-polarization components during the propagation, the cell's rubbing direction should be oriented at $0^{\circ}$ or $90^{\circ}$. The phase shifter could compensate a possible phase shift between two polarization components introduced by the rotator. When an electric field is applied across the LC layer, the directors become predominantly parallel to the electric field. The twisted structure is thus distorted, and, with voltage above threshold, begins to vanish. The polarization direction of the light is no longer rotated as light passes through the cell.

One of advantages of using the TN cell is that we do not need to know precise dependence of parameters of the cell on the applied voltage. In the transmitted light with use two rotators and can apply the same voltage to the both cell simultaneously using the same generator output. Typically the Freedericksz voltage threshold occurs at $2-3 \mathrm{~V}_{\text {rms }}$. In this case, the voltage $\sim 0.5 \mathrm{~V}_{\text {rms }}$ will set both rotators in the state $\mathrm{ON}$, and voltage $5 \mathrm{~V}_{\text {rms }}$ will put them in the state OFF.

It is promising to employ ferroelectric liquid crystal (FLC) cell in $90^{\circ}$ polarization rotator. They are known for their fast switching speed and bistability. The switching speed is very fast, which is usually a few microseconds. The two states are bistable, which means the electric field is not required once the tilting is finished [25,26].

Let us examine in more detail how the beam-shearing assembly works. For example, the initial ray $\mathrm{A}_{0}$ falling on the first prism DIC1 has coordinates $(0,0)$. Here the first number means a coordinate along the $\mathrm{X}$-axis, and the second one is the coordinate along the $\mathrm{Y}$-axis. The shear plane of the first DIC prism is parallel to the $\mathrm{X}$-axis. The prism deflects a ray with $\mathrm{Y}$-polarization in the negative direction by distance $-d / 2$ and shifts a ray with $\mathrm{X}$-polarization in the positive direction by the same amount. It creates a shear distance $d$ between the components along the $\mathrm{X}$-axis. Thus, the first output ray $\mathrm{A}_{1}$ has $\mathrm{X}$-polarization and coordinates $(d / 2,0)$. The $\mathrm{Y}$-polarized second ray $\mathrm{B}_{1}$ has coordinates $(-d / 2,0)$.

The $90^{\circ}$ polarization rotator preserves the linear polarizations of the beams $\mathrm{A}_{1}$ and $\mathrm{B}_{1}$ (state $\mathbf{O F F}$ ) or turns them by $90^{\circ}$ without altering the ray positions (state $\mathbf{O N}$ ). In the first case (left column) the output rays $\mathrm{A}_{2}$ and $\mathrm{B}_{2}$ are polarized along the $\mathrm{X}$-axis and $\mathrm{Y}$-axis, respectively. In the second case (right column) the rays $\mathrm{A}_{2}^{\prime}$ and $\mathrm{B}_{2}^{\prime}$ are polarized along the $\mathrm{Y}$-axis and $\mathrm{X}$-axis, correspondently.

The second prism DIC2 is oriented orthogonal to the direction of the first prism. So, the shear direction of the second prism lies along the Y-axis. The prism moves position of a ray with Y-polarization by distance $d / 2$ in the positive direction and deflects a ray with $\mathrm{X}$ polarization in the negative direction by the same amount.

First we consider a case, which is shown on the left side of Fig. 3, where the rotator state is $\mathbf{O F F}$, and therefore the ray polarizations are not changed. Then ray $\mathrm{A}_{2}$ with $\mathrm{X}$-polarization and coordinates $(d / 2,0)$ passes the second prism with negative displacement $-d / 2$ in the $\mathrm{Y}$ direction, and the output ray $\mathrm{A}_{3}$ has coordinates $(d / 2,-d / 2)$. In the meanwhile, the coordinates of ray $\mathrm{B}_{2}$ are changed by $d / 2$ in the positive direction along $\mathrm{Y}$-axis, and the coordinates for output ray $\mathrm{B}_{3}$ are $(-d / 2, d / 2)$. As a result, in the first case the dual beam that falls on the specimen has shear direction $-45^{\circ}$. The first case can be described by the following flow-chart: 


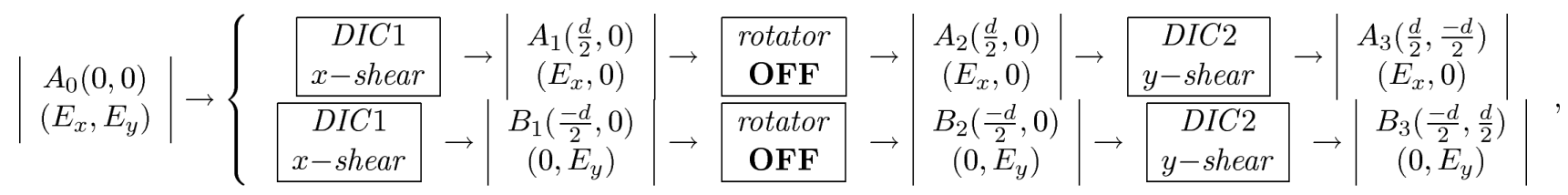

where $E_{X}$ and $E_{y}$ are the corresponding amplitudes of polarization components.

For the second case, after the rotator switches the polarizations by $90^{\circ}$, the ray $\mathrm{A}_{2}^{\prime}$ is linearly polarized along the $\mathrm{Y}$-axis. Therefore the second prism DIC2 changes Y-coordinate of the ray by $d / 2$, and the output ray $\mathrm{A}_{3}^{\prime}$ has coordinates $(d / 2, d / 2)$. Also the second prism deflects the X-polarized ray $\mathrm{B}_{2}^{\prime}$ by $-d / 2$, so that the second output ray $\mathrm{B}_{3}{ }_{3}$ has coordinates $(-d / 2,-d /$ 2 ). Hence, the output beam has a shear direction $+45^{\circ}$. This case can be illustrated by the next flow-chart:

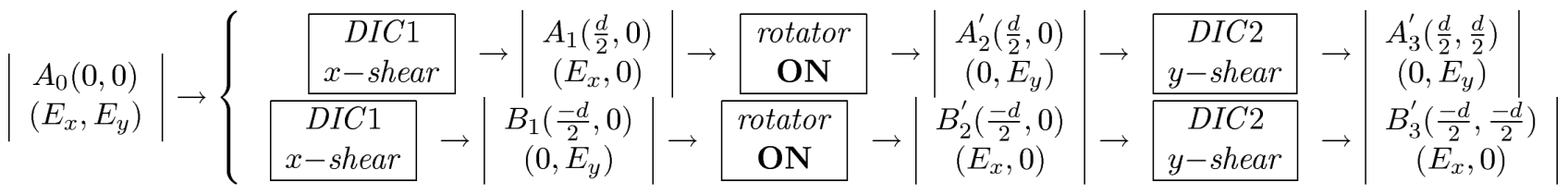

As one can see, the both outcome shears have the same amount, which equals to the shear $d$ introduced by a single DIC prism multiplied by $\sqrt{2}$. The shear vectors are mutually orthogonal and oriented at azimuths $\pm 45^{\circ}$. The median axis of two beams passes through point $(0,0)$ in the both cases. Therefore there is no misalignment between the images.

It is necessary to mention that switch of the light polarization between two DIC prisms affects the outcome bias (Fig. 3). Let's assume that prisms DIC1 and DIC2 introduce optical path differences $\Lambda_{1}$ and $\Lambda_{2}$ between beam components polarized parallel and perpendicular to the prism shear plane, respectively. If the rotator state is $\mathbf{O F F}$ then polarization of ray $\mathrm{A}_{1} \mathrm{~A}_{3}$ is parallel to the shear plane of the first DIC prism and perpendicular to the shear plane of the second prism. Thus, in the OFF state the beamshearing assembly introduces combined bias $\Lambda_{1}-\Lambda_{2}$. In the $\mathbf{O N}$ state the rotator switches polarizations by $90^{\circ}$, and polarization of ray $\mathrm{A}_{1} \mathrm{~A}_{3}^{\prime}$ is parallel to the shear planes of the both DIC prisms. In this case the combined bias is $\Lambda_{1}+\Lambda_{2}$.

\section{Data processing}

\subsection{Mathematical model of OI-DIC image}

A conventional DIC image can be modeled as the superposition of one image over an identical copy that is displaced in the object (specimen) plane by a small shear vector $\mathbf{d}$ and phase shifted by bias $\Lambda$ produced by two DIC prisms, which are located in the illumination and imaging beams correspondently. For simplicity, let's consider a phase non-birefringent specimen with two-dimensional distribution of optical path $\varphi(x, y)$. Here we use Cartesian coordinates $\mathrm{XOY}$ in the object plane. The specimen is illuminated by monochromatic light with wavelength $\lambda$. Intensity distribution $I(x, y)$ in the DIC image depends on the specimen orientation and varies proportionally with the scalar product of shear and optical path gradient vectors: 


$$
I(x, y)=\tilde{I} \sin ^{2}\left\{\frac{\pi}{\lambda}[\Lambda+\mathbf{d} \cdot \nabla \varphi(x, y)]\right\}+I_{c}(x, y),
$$

where $\tilde{I}$ is the initial beam intensity, $\nabla \varphi(x, y)$ is the optical path gradient vector, and $I_{\mathcal{c}}(x, y)$ corresponds to an offset of the intensity signal, which caused by the stray light.

It follows from the formula (2) that if the shear and optical path gradient vectors are parallel then their scalar product is maximal and the image contrast is maximal also. Where the shear vector is perpendicular to the gradient vector their scalar product is zero and the contrast equals zero correspondently. Thus regular DIC technique shows two-dimensional distribution of the optical path gradient encountered along the shear direction. It is therefore prudent to examine unknown objects at several azimuth orientations.

If $\mathrm{X}$-coordinate is parallel to the shear plane then shear vector $\mathbf{d}$ and gradient vector $\nabla \varphi(x$, y) could be presented in the expanded coordinate forms:

$$
\mathbf{d}=(d, 0)
$$

where $d$ is shear amount, and

$$
\nabla \varphi(x, y) \equiv\left(\frac{d \varphi(x, y)}{d x}, \frac{d \varphi(x, y)}{d y}\right)=(\gamma(x, y) \cos \theta(x, y), \gamma(x, y) \sin \theta(x, y))
$$

where $Y(x, y)$ is gradient magnitude and $\theta(x, y)$ is gradient azimuth. The gradient magnitude can be interpreted as product of refractive index gradient and optical section depth.

After scalar multiplication of the shear and gradient vectors we obtain the following equation in coordinate form for the regular DIC image:

$$
I(x, y)=\tilde{I} \sin ^{2}\left\{\frac{\pi}{\lambda}[\Lambda+d \cdot \gamma(x, y) \cos \theta(x, y)]\right\}+I_{c}(x, y) .
$$

As it is shown in Section 2, OI-DIC beam shearing assembly produces two orthogonal shears with shear amount equals to the shear introduced by a single DIC prism multiplied by $\sqrt{2}$. If X-coordinate is parallel to the shear plane of the first DIC prism (see Fig. 3) then shear vectors $\mathbf{d}_{i}$ could be written in the following coordinate form:

$$
\mathbf{d}_{i}=\left(d,(-1)^{i} d\right),
$$

where $i=1,2$ corresponds to the state $\mathrm{OFF}$ or $\mathrm{ON}$ of $90^{\circ}$ polarization rotator (first or second state of shear direction).

Another peculiarity of OI-DIC beam shearing assembly is that the bias introduced by its internal DIC prism is subtracted when the polarization rotator is in OFF state or they are added when the polarization rotator is in ON state. In case of the transmitted light microscope (Fig. 1) the combined bias $\Lambda_{\Sigma i}$ would be determined by the formula:

$$
\Lambda_{\sum_{i}}=\Lambda_{1}+\Lambda_{4}+(-1)^{i}\left(\Lambda_{2}+\Lambda_{3}\right),
$$


where $\Lambda_{1}, \Lambda_{2}, \Lambda_{3}$, and $\Lambda_{4}$ are biases contributed by prisms DIC1, DIC2, DIC3, and DIC4, respectively.

The combined bias of the reflected light setup (Fig. 2) is described by similar equation:

$$
\Lambda_{\sum i}=2 \Lambda_{1}+(-1)^{i} \Lambda_{2}
$$

Substituting (4) and (6) into (2), and taking into account variable bias $\Gamma$ created by the phase shifter and combined bias $\Lambda_{\Sigma i}$ introduced by DIC prisms (see formulae $(7,8)$, we readily find equation describing OI-DIC microscope:

$$
I_{i}(x, y)=\tilde{I} \sin ^{2}\left\{\frac{\pi}{\lambda}\left[\Gamma+\Lambda_{\sum_{i}}+\sqrt{2} d \cdot \gamma(x, y) \cos \left(\theta(x, y)-(-1)^{i} \frac{\pi}{4}\right)\right]\right\}+I_{c}(x, y)
$$

where note $i=1,2$ corresponds to the $1^{\text {st }}$ or $2^{\text {nd }}$ state of shear direction, $-45^{\circ}$ or $+45^{\circ}$ correspondently.

\subsection{Optimal bias}

In order to achieve the best DIC picture it is necessary to apply the optimal bias. For example, we have an extremely shallow depression or elevation that introduces small optical path difference between the studied structure and the media. The high bias would create a bright background and the observed structure would be hardly visible. If bias is low the image background field becomes dark gray and yields very high sensitivity, bringing out image regions with minute phase differences. This conclusion is in agreement with data obtained by Salmon and Tran [28], who indicate that the best contrast is achieved when bias is adjusted to extinguish the light coming from one edge of the object of interest. This occurs when bias equals optical path difference introduced by the edge with the opposite sign. Salmon and Tran found that for the edges of organelles and cells, the optical path difference corresponds to about $N 10^{\text {th }}$ the wavelength or greater, but for microtubules and tiny organelles in cell, optical path difference is very small, less than $1 / 100^{\text {th }}$ the wavelength of green light. They recommend using about $1 / 15^{\text {th }}-1 / 20^{\text {th }}$ the wavelength bias for observation of microtubules in order to have sufficient light at the camera. A similar result was found by Schnapp [29].

The bias optimization can be explored with using equation (5). Let consider a simplified sample with a binary gradient magnitude distribution such that a half of the sample has a gradient magnitude of $+\gamma$ and the other half has a phase difference of $-\gamma$. The shear and gradient directions of the sample are parallel $\left(\theta=0^{\circ}\right)$.

We expect the best results to be achieved when DIC images have the highest contrast C:

$$
C=\frac{I_{\max }-I_{\min }}{I_{\max }+I_{\min }},
$$

where $I_{\max }$ and $I_{\min }$ are the maximal and minimal intensities in the image.

The maximal and minimal intensities can be determined using equation (5). For the specimen under consideration the contrast of DIC image is the following: 


$$
C=\frac{\sin \left(\frac{2 \pi}{\lambda} \Lambda\right) \sin \left(\frac{2 \pi}{\lambda} \gamma d\right)}{1-\cos \left(\frac{2 \pi}{\lambda} \Lambda\right) \cos \left(\frac{2 \pi}{\lambda} \gamma d\right)+\frac{2 I_{c}}{\tilde{I}}} .
$$

The ratio $I_{\mathcal{C}} / \tilde{I}$ is called the extinction ratio. Derivative $d C / d \Lambda$ of this equation is the next:

$$
\frac{d C}{d \Lambda}=\sin \left(\frac{2 \pi}{\lambda} \gamma d\right) \frac{\left(1+\frac{2 I_{c}}{\tilde{I}}\right) \cos \left(\frac{2 \pi}{\lambda} \Lambda\right)-\cos \left(\frac{2 \pi}{\lambda} \gamma d\right)}{1-\cos \left(\frac{2 \pi}{\lambda} \Lambda\right) \cos \left(\frac{2 \pi}{\lambda} \gamma d\right)+\frac{2 I_{c}}{\tilde{I}}}
$$

The derivative is zero at $\Lambda \approx \gamma \cdot d$. Thus, the maximal contrast is achieved when bias equals optical path difference in the sample.

According to formula (11) the contrast of microtubules and tiny organelles with phase difference $N 100$ equals 0.38 at bias $N 20$ and the stray light in the system is absent, 0.27 at bias $N 20$ and extinction ratio100, 0.06 at bias $N 4$ and both extinctions. The contrast at the small bias is more 6 times the one at the large bias. But it is very important to have a high extinction in order to effectively use a small bias for studying tiny structures. The contrast of cell walls and organelle edges with phase difference $N 10$ is 0.79 at bias $N / 20$ and infinity extinction, 0.72 at bias $N / 20$ and extinction 100 , and 0.59 at bias $N / 4$ and the both extinctions.

In addition to reducing contrast the low extinction decreases the dynamic range of measurement and can cause a diffraction anomaly in the Airy pattern. The lower extinction is a significant problem in microscopes equipped with high numerical-aperture lenses. To reduce the beam depolarization we can use a polarization rectifier [30,31].

\subsection{Algorithm for mapping two-dimensional gradient vector field with using low bias swing}

The contrast in DIC image is generated in very thin optical section of the specimen. Estimated thickness of the section could be about few tenths of micron in case of employing a high NA objective lens. Additional contrast reduction occurs if the high-resolution DIC prisms with small shear amount are exploited. Taking into account that the maximal difference between refractive indices of cell structures and surrounding water media is 0.2 the maximal optical path difference would be about $0.1 \mu \mathrm{m}$ for the most biological specimens. This number corresponds to $1 / 5^{\text {th }}$ the wavelength of green light. Actually, in the most cases, the introduced optical path difference is significantly less of that. Therefore it would be beneficial to use a low bias for obtaining the best pictures.

This approach requires preliminary alignment of the OI-DIC system in order to work near the light extinction. At first, it is necessary to set the internal DIC prisms in such position that they mutually compensate or do not introduce the bias, $\Lambda_{2}+\Lambda_{3}=0$ or $\Lambda_{2}=0$ (see formulas (7) and (8), respectively). This allows to eliminate the bias that depends on shear direction. Next, using the external DIC prisms and/or the phase shifter, we have to achieve the darkest background in the image. That means the total bias is zero, $\Gamma+\Lambda_{\Sigma}=0$.

In order to find the two-dimensional distribution of the gradient magnitude and azimuth $Y$ and $\theta$ we capture two sets of raw DIC images at shear directions $-45^{\circ}$ and $+45^{\circ}$ with negative, zero and positive biases: $-\Gamma_{0}, 0$, and $+\Gamma_{0}[18]$. The following group of equations represents these six DIC images: 


$$
I_{i, j}(x, y)=\tilde{I} \sin ^{2}\left\{\frac{\pi}{\lambda}\left[j \Gamma_{0}+\sqrt{2} d \gamma(x, y) \cos \left(\theta(x, y)-(-1)^{i} \frac{\pi}{4}\right)\right]\right\}+I_{c}(x, y)
$$

where $j=-1,0,1$.

Initially two terms are computed $(i=1,2)$ :

$$
A_{i}(x, y)=\frac{I_{i, 1}(x, y)-I_{i,-1}(x, y)}{I_{i, 1}(x, y)+I_{i,-1}(x, y)-2 I_{i, 0}(x, y)} \tan \left(\frac{\pi \Gamma_{0}}{\lambda}\right)
$$

Using equations (13) we can show that

$$
\begin{aligned}
& A_{1}(x, y)=\tan \left(\frac{2 \sqrt{2} \pi}{\lambda} d \gamma(x, y) \cos \left(\theta(x, y)+\frac{\pi}{4}\right)\right), \\
& A_{2}(x, y)=\tan \left(\frac{2 \sqrt{2} \pi}{\lambda} d \gamma(x, y) \sin \left(\theta(x, y)+\frac{\pi}{4}\right)\right),
\end{aligned}
$$

Using the obtained terms we can calculate the quantitative two-dimension distributions of the gradient magnitude and azimuth of optical paths in the specimen as:

$$
\begin{gathered}
\gamma(x, y)=\frac{\lambda}{2 \sqrt{2} \pi d} \sqrt{\sum_{i=1}^{2} \arctan ^{2}\left[A_{i}(x, y)\right]} \\
\theta(x, y)=\arctan \left(\frac{\arctan A_{2}(x, y)}{\arctan A_{1}(x, y)}\right)-\frac{\pi}{4}
\end{gathered}
$$

The gradient magnitude represents increment of the optical path difference, which is in nanometers, along lateral coordinate, which is also in nanometers. Thus, the gradient magnitude is unitless. The shear amount $d$ can be measured as it is described in Appendix A or found in Table 1.

Notice that the algorithm considered above employs ratios between intensities of light that have interacted with the specimen. Therefore it suppresses contributions of absorption by the specimen or from nonuniformity of illumination, which can otherwise deteriorate a DIC image.

\subsection{Algorithm for mapping two-dimensional gradient vector field with using $\pi / 4$ phase step}

If the maximal optical path difference exceeds $1 / 5^{\text {th }}$ the wavelength we can also employ other algorithms, which are similar to one used in phase-shifting interferometers [32]. For example, let's consider the modification of algorithm using four frame of intensity data recorded with phase shifts of $0, \pi / 2, \pi$, and $3 \pi / 2$. This algorithm is common in the interference microscopy [6]. Similar approach we proposed for mapping birefringence at the normal reflection [33].

Under these conditions, the intensity distribution $I_{i j}(x, y)$ on the CCD camera is

$$
I_{i, j}(x, y)=\tilde{I} \sin ^{2}\left\{\frac{j \pi}{4}+\frac{\sqrt{2} \pi d \gamma(x, y)}{\lambda} \cos \left(\theta(x, y)-(-1)^{i} \frac{\pi}{4}\right)\right\}+I_{c}(x, y) \text {. }
$$


where $j=0,1,2,3$ conforms to four biases, and $i=1,2$ corresponds to states OFF and ON of the polarization rotators. Here we took into account that phase step $\pi / 2$ is created by bias step $N 4$.

Next two terms are calculated:

$$
\tilde{A}_{i}(x, y)=\frac{I_{i, 3}(x, y)-I_{i, 1}(x, y)}{I_{i, 0}(x, y)-I_{i, 2}(x, y)}
$$

Using equations (17) we have

$$
\begin{aligned}
& \tilde{A}_{1}(x, y)=\tan \left(\frac{2 \sqrt{2} \pi}{\lambda} d \gamma(x, y) \cos \left(\theta(x, y)+\frac{\pi}{4}\right)\right) \\
& \tilde{A}_{2}(x, y)=\tan \left(\frac{2 \sqrt{2} \pi}{\lambda} d \gamma(x, y) \sin \left(\theta(x, y)+\frac{\pi}{4}\right)\right)
\end{aligned}
$$

Finally we obtain the quantitative two-dimension distributions of the gradient magnitude and azimuth of optical paths:

$$
\begin{gathered}
\gamma(x, y)=\frac{\lambda}{2 \sqrt{2} \pi d} \sqrt{\sum_{i=1}^{2} \arctan ^{2}\left[\tilde{A}_{i}(x, y)\right]}, \\
\theta(x, y)=\arctan \left(\frac{\arctan \tilde{A}_{2}(x, y)}{\arctan \tilde{A}_{1}(x, y)}\right)-\frac{\pi}{4}
\end{gathered}
$$

The choice of the algorithms with low bias swing or $\pi / 4$ step depends on the optical path difference range of the specimen under investigation. The first algorithm is more sensitive for specimen with low optical path difference. It could be optimized to get the best picture by using swing that equals to the maximal optical path difference. The second algorithm does not require preliminary estimation of the optical path difference range. It works well with large optical path difference. The researcher could start with the second algorithm in order to estimate the specimen optical path difference and then apply the first algorithm with optimized swing amount.

\subsection{Correction of background gradient vector field}

When measuring a specimen with small gradient magnitude, it is important to minimize errors that are due to background gradient vector field across the image. Lenses, DIC prisms, $\mathrm{LC}$ rotators and phase shifter contribute to the background. The spatially varying background gradient vector field leads to systematic error in the measurement of specimen gradient magnitude and azimuth.

For correction of the background gradient vector field it is possible to employ special procedure, which mathematical description we published early [34, 35]. The procedure is based on specimen images, which are recorded with no structures in the optical path. In order to record the background set of images the object is moved out of the camera view field by use of the $x-y$ translator of the microscope. If empty area around the object is not available one could utilize a defocused picture of the object as the background. The background images are captured at the same beam-shearing assembly settings as for the object itself. Using these images we compute terms $A_{b g i}(x, y)$ or $\tilde{A}_{b g i}(x, y)$ according to formulae (14) or (18). Then the differences between the terms $A_{i}(x, y)-A_{b g i}(x, y)$ or $\tilde{A}_{i}(x$, $y)-\tilde{A_{b g i}}(x, y)$ are used in modified formulas (16) or (20) for determining gradient magnitude and azimuth: 


$$
\begin{gathered}
\gamma(x, y)=\frac{\lambda}{2 \sqrt{2} \pi d} \sqrt{\sum_{i=1}^{2} \arctan ^{2}\left[A_{i}(x, y)-A_{b g i}(x, y)\right]} \\
\theta(x, y)=\arctan \left(\frac{\arctan \left[A_{2}(x, y)-A_{b g 2}(x, y)\right]}{\arctan \left[A_{1}(x, y)-A_{b g 1}(x, y)\right]}\right)-\frac{\pi}{4},
\end{gathered}
$$

or

$$
\begin{gathered}
\gamma(x, y)=\frac{\lambda}{2 \sqrt{2} \pi d} \sqrt{\sum_{i=1}^{2} \arctan ^{2}\left[\tilde{A}_{1}(x, y)-\tilde{A}_{b g i}(x, y)\right]} \\
\theta(x, y)=\arctan \left(\frac{\arctan \left[\tilde{A}_{2}(x, y)-\tilde{A}_{b g 2}(x, y)\right]}{\arctan \left[\tilde{A}_{1}(x, y)-\tilde{A}_{b g 1}(x, y)\right]}\right)-\frac{\pi}{4}
\end{gathered}
$$

In addition to correcting background gradient field of the specimen under investigation this procedure also accounts for slight miscalibration of polarization states of the beam-shearing assemblies.

Typically, the background retardance does not change considerably over a time period of many minutes. Therefore the intermediate results $A_{b g i}(x, y)$ or $\tilde{A}_{b g i}(x, y)$ can be employed repeatedly to correct, e.g., a time series of images measuring the subtle changes in optical path gradient in a live specimen.

It is possible that intensity of the illumination would fluctuate during the time series image acquisition. We observed such fluctuations using high power mercury arc lamps. Also retardance of the LC phase shifter could slightly vary because of a temperature change. These reasons of an error in the measured specimen gradient vector distribution cannot be corrected with the described background correction procedure. But this small error gradient would be uniform across the image. Therefore if there is an image area with known gradient vector we can use it for additional correction.

For example, the obtained image shows some gradient magnitude $\gamma_{\text {cor }}$ and azimuth $\theta_{c o r}$ in an image point where the gradient is expected to be zero. This allows us to compute the correction terms $A_{c o r i}$ or $\tilde{A}_{c o r i}$, where $i=1,2$, using equations (15) or (19), respectively:

$$
\begin{aligned}
& A_{c o r} 1=\tilde{A}_{c o r 1}=\tan \left(\frac{2 \sqrt{2} \pi}{\lambda} d \gamma_{c o r} \cos \left(\theta_{c o r}+\frac{\pi}{4}\right)\right), \\
& A_{c o r 2}=\tilde{A}_{c o r 2}=\tan \left(\frac{2 \sqrt{2} \pi}{\lambda} d \gamma_{c o r} \sin \left(\theta_{c o r}+\frac{\pi}{4}\right)\right) .
\end{aligned}
$$

Then the obtained terms are applied for calculating the corrected gradient magnitude and azimuth for the entire image:

$$
\begin{gathered}
\gamma(x, y)=\frac{\lambda}{2 \sqrt{2} \pi d} \sqrt{\sum_{i=1}^{2} \arctan ^{2}\left[A_{i}(x, y)-A_{b g i}(x, y)-A_{\text {cor } i}\right]} \\
\theta(x, y)=\arctan \left(\frac{\arctan \left[A_{2}(x, y)-A_{b g 2}(x, y)-A_{\text {cor } 2}\right]}{\arctan \left[A_{1}(x, y)-A_{b g 1}(x, y)-A_{\text {cor } 1}\right]}\right)-\frac{\pi}{4}
\end{gathered}
$$

or 


$$
\begin{gathered}
\gamma(x, y)=\frac{\lambda}{2 \sqrt{2} \pi d} \sqrt{\sum_{i=1}^{2} \arctan ^{2}\left[\tilde{A}_{i}(x, y)-\tilde{A}_{b g i}(x, y)-\tilde{A}_{c o r} i\right]} \\
\theta(x, y)=\arctan \left(\frac{\arctan \left[\tilde{A}_{2}(x, y)-\tilde{A}_{b g 2}(x, y)-\tilde{A}_{c o r 2}\right]}{\arctan \left[\tilde{A}_{1}(x, y)-\tilde{A}_{b g 1}(x, y)-\tilde{A}_{c o r 1}\right]}\right)-\frac{\pi}{4}
\end{gathered}
$$

The formulas (24) and (25) allow to receive gradient images, which are corrected on the background retardance, illumination intensity fluctuations, LC retardance variations and systematic errors.

After correction of the gradient vector field, we can compute an enhanced regular DIC image with any shear direction, shear amount and bias retardation using formula (5) with zero offset term. The computed image will be pure phase gradient image with less noise and without deterioration caused by absorption, illumination nonuniformity and light scattering.

\subsection{Computation of optical path image}

The obtained optical path gradient vector field allows us to compute the corresponding twodimensional scalar field, the optical path, by using numerical integration. The gradient field is a conservative vector field; the computed optical path depends only on the endpoints of that integral, not the particular integration route taken.

Many computation techniques could be employed for determining the optical path, for instance, line integration [16, 36], iterative method [37], various modifications of Fourier integration [11-15, 20, 38, 39], extended Fried algorithm [40], etc. In principle, any of these methods could be employed with us of our OI-DIC microscope. We shall not compare them in this current publication because it expands its size substantially. So it will be done in a separate article.

We use the optical path computation, developed by D. Biggs (KB Imaging Solution LLC, Loomis, CA), which is based on iterative deconvolution approach. This technique employs the same principles as deconvolution techniques normally used to remove out-of-focus haze [41-43].

\section{Experimental verification}

The first experimental setup in the transmitted mode, which is shown in Fig.1, was implemented on an upright microscope Olympus BX-61 (Olympus America, Center Valley, PA). The beam-shearing assemblies employed two pairs of regular DIC prisms in their original mounts and $2.2 \mathrm{~mm}$-thick liquid crystal $90^{\circ}$ polarization rotators in custom made 7 mm-thick holders. The axial displacement of the outside prism DIC4 in the imaging path was $32 \mathrm{~mm}$ up, and the axial displacement of the outside prism DIC1 in the illumination path was $16 \mathrm{~mm}$ down. The internal prisms DIC2 and DIC3 were placed in the originally designed positions. We made single displacement compensator, which corrects misalignment in the interfering beams caused by displacements of the both outside prisms. The LC optics was made by Boulder Vision Optics (Boulder, CO). The images were captured by a monochromatic CCD camera Infinity 3-1M (Lumenera, Canada). Using Matlab $^{\text {TM }}$ (The MathWorks, Inc., Natick, MA) we developed software for setup control and image processing.

Figure 4 shows OI-DIC images of the hematoxylin-eosin (H\&E) stained breast cancer tissue sample, which contains collagenous stroma collagen surrounding the nests of cancer cells. The pictures were taken with using 40×/0.75P UPlanFL objective lens and 546/40nm bandpath filter. The picture size is $186 \mu \mathrm{m} \times 145 \mu \mathrm{m}$. We employed two crossed high- 
resolution DIC prisms U-DICTHR in the top beam-shearing assembly. According to Table 1 the shear distance of single prism equals $0.18 \mu \mathrm{m}$. Then the shear distance of the crossed prism pair is $0.25 \mu \mathrm{m}(0.18 \mu \mathrm{m}$ multiplied by $\sqrt{2})$. The bias modulation amplitude was $0.15 \lambda$.

Six raw DIC images $\boldsymbol{I}_{i, j}(x, y)$ are shown on the left, where $i=1$ and $i=2$ correspond to the Xand $\mathrm{Y}$ - shear direction, respectively. Notations $j=-1,0,1$ conform to bias $-0.15 \lambda, 0$ or $0.15 \lambda$. All DIC images were captured within $1 \mathrm{sec}$. In white light the specimen is mostly red. If there is a green filter in the optical path then many specimen structures become dark due to significant absorption in the green-blue spectral region. So the shadow cast DIC features are hardly visible. But they are present, and the computer processing produces quantitative gradient magnitude and azimuth maps (shown in the center). Further computation allows to receive the phase image and regular DIC picture with drastically improved clarity. The enhanced DIC image could be computed with any shear direction and amount without need to rotate either specimen or DIC prisms. The shown enhanced DIC image was computed with the horizontal shear direction, for instance. It could be compared with regular DIC image with the same shear direction, which is displayed on the left top.

Figure 5 shows example of the phase OI-DIC image of a live crane fly spermatocyte during metaphase of meiosis I taken with a Olympus UPlanFl 100×/1.30 oil immersion objective lens and 546/30nm interference filter. Image size is $68 \mu \mathrm{m} \times 68 \mu \mathrm{m}$. The image acquisition and processing took about 1 second each. The three autosomal bivalent chromosomes are in sharp focus at the spindle equator, along with one of the X-Y sex univalents, which is located on the right. The tubular distribution of mitochondria surrounding the spindle is clearly evident. Both polar flagella in the lower centrosome are in focus, appearing as a letter "L" lying on its side. The experiment was done together with Prof. LaFountain (State University of New York, Buffalo, NY). The phase image was computed by Dr. Biggs (KB Imaging Solution LLC, Loomis, CA) with employing iterative deconvolution approach mentioned above.

\section{Conclusion}

Here we are proposing new quantitative orientation-independent DIC microscope with high speed switching shear direction and bias modulation. The microscope does not require any mechanically rotating the specimen or the prisms. Therefore there is no image misalignment. All components do not require a special design and are available on the market. We proposed techniques for measuring parameters of DIC prisms and calibrating the bias. Two sets of raw DIC images at the orthogonal shear directions are captured and processed within a second. Then the quantitative image of optical path gradient distribution within a thin optical section is computed. The obtained data are also used to calculate the quantitative distribution of optical phase, which represents refractive index gradient or height distribution. It is possible to generate back the enhanced regular DIC images with any desired shear direction.

\section{Acknowledgments}

The author thanks David Biggs of KB Imaging Solution LLC (Loomis, CA) for helpful discussion and providing software for computing phase images and Richard Langill of the Marine Biological Laboratory (Woods Hole, MA) for hardware development. We are also grateful to Shinya Inoué of the MBL, and to James LaFountain of State University of New York (Buffalo, NY) for their encouragement and support. This publication was made possible by Grant Number R01-GM101701 from the National Institute of General Medical Sciences, National Institutes of Health. Its contents are solely the responsibility of the author and do not necessarily represent the official views of the National Institute of General Medical Sciences or the National Institutes of Health. 


\section{References}

1. Smith, FH. Interference microscope. US Patent. 2,601,175. Aug 5. 1947

2. Smith FH. Microscopic interferometry. Research (London). 1955; 8:385-395.

3. Nomarski, G. Interferential polarizing device for study of phase object. US Patent. 2924142. May 14. 1952

4. Allen RD, David GB, Nomarski G. The Zeiss-Nomarski differential equipment for transmitted light microscopy. Zeitschrift für Wissenschaftliche Mikroscopie und Mickroskopische Technik. 1969; 69(4):193-221.

5. Inoué, S. Ultrathin optical sectioning and dynamic volume investigation with conventional light microscopy. In: Stevens, J., editor. Three-Dimensional Confocal Microscopy: Volume Investigation of Biological Systems. Academic Press; 1994. p. 397-419.

6. Oldenbourg, R.; Shribak, M. Microscopes. In: Bass, M., editor. Handbook of Optics, Third Edition, Volume I: Geometrical and Physical Optics, Polarized Light, Components and Instruments. McGraw-Hill; 2010. p. 28.1-28.62.

7. Holzwarth GM, Webb SC, Kubinski DJ, Allen NS. Improving DIC microcopy with polarization modulation. J Microscopy. 1997; 188(Pt 3):249-254.

8. Holzwarth GM, Hill DB, McLaughlin EB. Polarization-modulated differential-interference contrast microscopy with a variable retarder. Applied Optics. 2000; 39:6288-6294. [PubMed: 18354637]

9. Ooki H, Iwasaki Y, Iwasaki J. Differential interference contrast microscope with differential detection for optimizing image contrast. Applied Optics. 1996; 35:2230-2234. [PubMed: 21085356]

10. Hariharan P, Roy M. Achromatic phase-shifting for two-wavelength phase- stepping interferometry. Optics Communication. 1996; 126:220-222.

11. Arnison MR, Larkin KG, Sheppard CJR, Smith NI, Cogswell CJ. Linear phase imaging using differential interference contrast microscopy. J Microscopy. 2004; 214(Pt 1):7-12.

12. King SV, Libertun AR, Preza C, Cogswell CJ. Calibration of a phase-shifting DIC microscope for quantitative phase imaging. Proc SPIE. 2007; 6443:64430M.

13. Ishiwata $\mathrm{H}$, Itoh $\mathrm{M}$, Yatagai $\mathrm{T}$. A new method of three-dimensional measurement by differential interference contrast microscope. Optics Communications. 2006; 260:117-126.

14. Ishiwata $\mathrm{H}$, Itoh $\mathrm{M}$, Yatagai $\mathrm{T}$. A new analysis for extending the measurement range of the retardation-modulated differential interference contrast (RM-DIC) microscope. Optics Communications. 2008; 281:1412-1423.

15. Noguchi A, Ishiwata H, Itoh M, Yatagai T. Optical sectioning in differential interference contrast microscopy. Optics Communications. 2009; 282:3223-3230.

16. Shribak M, Inoué S. Orientation-independent differential interference contrast microscopy. Applied Optics. 2006; 45:460-469. [PubMed: 16463729]

17. Shribak, M. Orientation-independent differential interference contrast microscopy technique and device. US Patent. 7,564,618. Dec 17. 2003

18. Shribak, M. Orientation-independent differential interference contrast microscopy technique and device. US Patent. 7,233,434. Dec 17. 2003

19. Fabre L, Inoue Y, Aoki T, Kawakami S. Differential interference contrast microscope using photonic crystals for phase imaging and three-dimensional shape reconstruction. Appl Opt. 2009; 48:1347-1357. [PubMed: 19252636]

20. Preza C, Snyder DL, Conchello JA. Theoretical development and experimental evaluation of imaging models for differential-interference-contrast-microscopy. J Opt Soc Am A. 1999; 16:2185-2199.

21. Hogan H. Getting the small picture. Photonics Spectra. 2003; 37(4):58-64.

22. Danz R, Gretscher P. C-DIC: a new microscopy method for rational study of phase structures in incident light arrangement. Thin Solid Films. 2004; 462-463:257-62.

23. Robinson, M.; Chen, J.; Sharp, G. Polarization Engineering for LCD Projection. Wiley; 2005. 
24. Chipman, RA. Polarimetry. In: Bass, M., editor. Handbook of Optics, Third Edition, Volume I: Geometrical and Physical Optics, Polarized Light, Components and Instruments. McGraw-Hill; 2010. p. 15.1-15.46.

25. Scharf, T. Polarized Light in Liquid Crystals and Polymers. Wiley; 2007.

26. Lueder, E. Liquid Crystal Displays: Addressing Schemes and Electro-Optical Effects. Wiley; 2010.

27. Gauza, S.; Wu, S-T. Liquid Crystals. In: Bass, M., editor. Handbook of Optics, Third Edition, Volume V: Atmospheric Optics, Modulators, Fiber Optics, X-Ray And Neutron Optics. McGrawHill; 2010. p. 8.1-8.40.

28. Salmon ED, Tran P. High resolution video-enhanced differential-interference contrast (VE-DIC) light microscopy. Meth Cell Biol. 1998; 56:153-184.

29. Schnapp BJ. View single microtubules by video light microscopy. Meth Enzymol. 1986; 134:561573. [PubMed: 2881191]

30. Shribak M, Inoué S, Oldenbourg R. Rectifiers for suppressing depolarization caused by differential transmission and phase shift in high NA lenses. Proc SPIE. 2001; 4481:163-174.

31. Shribak M, Inoué S, Oldenbourg R. Polarization aberrations caused by differential transmission and phase shift in high NA lenses: theory, measurement and rectification. Opt Eng. 2002; 41:943954.

32. Hariharan, P. Optical Interferometry. 2. Academic Press; London, United Kingdom: 2003.

33. Shribak M, Otani Y, Yoshizawa T. Autocollimation polarimeter for measuring two-dimensional distribution of birefringence. Optics and Spectroscopy. 2000; 89:155-159.

34. Shribak M, Oldenbourg R. Techniques for fast and sensitive measurements of two-dimensional birefringence distributions. Appl Opt. 2003; 42:3009-3017. [PubMed: 12790452]

35. Shribak M. Complete polarization state generator with one variable retarder and its application for fast and sensitive measuring of two-dimensional birefringence distribution. J Opt Soc Am A. $2011 ; 28: 410-419$.

36. Heise B, Sonnleitner A, Klement EP. DIC image reconstruction on large cell scans. Microscopy Research and Technique. 2005; 66:312-320. [PubMed: 16003783]

37. Preza C. Rotational-diversity phase estimation from differential-interference-contrastmicroscopy images. J Opt Soc Am A. 2000; 17:415-424.

38. van Munster EB, van Vliet LJ, Aten JA. Reconstruction of optical pathlength distributions from images obtained by a wide-field differential interference contrast microscope. J Microscopy. 1997; 188:149-157.

39. Yu SK, Liu TK, Lin SC. Height measurement of transparent objects by adopting differential interference contrast technology. Appl Opt. 2010; 49:2588-2596.

40. Prahl SA, Dayton A, Juedes K, Sanchez EJ, Lopez RP, Duncan DD. Experimental validation of phase using Nomarski microscopy with an extended Fried algorithm. J Opt Soc Am A. 2012; 29:2104-2109.

41. Holmes, TJ.; Bhattacharyya, S.; Cooper, JA.; Hanzel, D.; Krishnamurthi, V.; Lin, W.; Roysam, B.; Szarowski, DH.; Turner, JN. Light microscopic images reconstructed by maximum likelihood deconvolution. In: Pawley, JB., editor. Handbook of Biological Confocal Microscopy. Plenum Press; 1995. p. 389-402.

42. Biggs D, Andrews M. Acceleration of iterative image restoration algorithms. Appl Opt. 1997; 36:1766-1775. [PubMed: 18250863]

43. Shribak M, LaFountain J, Biggs D, Inoué S. Orientation-independent differential interference contrast microscopy and its combination with an orientation-independent polarization system. Journal of Biomedical Optics. 2008; 13:014011. [PubMed: 18315369]

44. Mehta SB, Sheppard CJR. Sample-less calibration of the differential interference contrast microscope. Applied Optics. 2010; 49:2954-2968. [PubMed: 20490258]

45. Müller CB, Weiß K, Richtering W, Loman A, Enderlein J. Calibrating differential interference contrast microscopy with dual-focus fluorescence correlation spectroscopy. Optics Express. 2008; 16:4322-4329. [PubMed: 18542529]

46. Duncan DD, Fischer DG, Dayton A, Prahl SA. Quantitative Carré differential interference contrast microscopy to assess phase and amplitude. J Opt Soc Am A. 2011; 28:1297-1306. 
47. Shribak, MI. Device for measuring birefringence of reflecting optical data carrier. USSR Patent 1,414,097. Mar 17. 1986

48. Shribak MI. A compensation method for measuring birefringence. Sov J Opt Technol. 1993; 60:546-549.

49. Shribak MI. Autocollimating detectors of birefringence. Proc SPIE. 1996; 2782:805-813.

50. Hartshorne, NH.; Stuart, A. Crystals and the Polarizing Microscope. 4. Edward Arnold; London, United Kingdom: 1970.

51. Shribak MI. Polarization separation of the forward and reverse beams in the reading of reflective carriers of information. Sov J Opt Technol. 1986; 53:389-391.

\section{Appendix A: Measuring shear distance}

Shear amount (distance) is the critical parameter of a DIC microscope that determines its contrast, sensitivity, resolution and optical section depth. Another issues with DIC microscopy is that, to derive quantitative information, one must know the amount of image shear. Generally, however, microscope manufacturers do not make that information available. As a result, one must measure this parameter. Munster et al. [38] determined the lateral shift by measuring the distance between the center of the bright spot and the center of the dark spot in image of a submicroscopic transparent latex sphere with bias set at $\pi / 2$. Mehta and Sheppard [44] measured shear by studying intensity distribution in the back focal plane of the microscope objective lens. Müller et al. [45] used a combination of fluorescence correlation spectroscopy and dynamic light scattering to determine shear. Duncan et al. [46] described a measurement setup with using a standard optical wedge.

The described below technique for determining shear distance is faster and more accurate than mentioned. We measure a shear angle and then compute the shear amount with employing formula (1), as it is explained in the end of this section.

In order to find the shear angle of DIC prism we do not need to know how the passing beam is transformed inside the prism. DIC prism splits an incident monochromatic plane wave into two orthogonally polarized plane wave with slightly different direction of their wavefronts. Fig. 6 illustrates splitting the incident linearly polarized beam into two separate output beams with shear angle $\epsilon$. Here the shear plane is parallel to the $X$-axis. As one can see from the picture, the shear angle $\epsilon$ (in radians) equals to derivative of the optical path difference (bias) $\Lambda$ with respect to the coordinate $x$ :

$$
\varepsilon=\frac{d \Lambda}{d x} .
$$

The optical path difference $\Lambda$ is connected with retardance $\delta$, written in degrees, and wavelength $\lambda$ in the simple way:

$$
\Lambda=\frac{\delta}{360^{\circ}} \lambda .
$$

Thus, the shear angle could be found by measuring a derivative of the retardance with respect to the coordinate along the shear direction:

$$
\varepsilon=\frac{\lambda}{360^{\circ}} \frac{d \delta}{d x}
$$


Retardance can be determined with a number of various techniques. In particularly, one could employ the return-path compensation method, which we proposed in 1986 [47-49]. This simple technique is similar to the Senarmont compensator [50]. Because of superposition of the probe and reverse beams the return-path arrangement has the following advantages: (i) owing to a double pass of a beam through a specimen under investigation, the sensitivity threshold is decreased by factor of two; (ii) the same optical elements are utilized twice, in the direct and the reverse beams. It makes the measurement set-up less expensive considerably, which is very important, because one high quality optical polarization element could cost several thousands dollars.

Schematic of the return-path setup is shown in Fig. 7. The device operates as follows. A laser radiates the collimated narrow beam, which is unpolarized or circularly polarized. The beam passes sequentially through a small hole in a screen, Glan-Thompson polarization beam splitter, quarter-wave plate QWP, and DIC prism. The DIC prism under investigation is placed on a linear stage with Vernier micrometer. The stage allows to move the prism in the horizontal direction. The beam passes through the quarter-wave plate and DIC prism two times being reflected from a mirror. The beam splitter deflects the orthogonal polarization component toward a screen. The minimum intensity of the deflected laser beam was visually determined by observing the laser spot brightness on the screen. It is also possible to use a photodiode PD to measure the beam intensity. The intensity of the radiation flux of the deflected orthogonal component $I$ is described by the following formula [51]:

$$
I^{\prime}=I\{\cos \delta \sin 2(\zeta-\chi)-\sin \delta \cos 2(\zeta-\chi) \sin 2(\zeta-\psi)\}^{2},
$$

where $I$ is the probing beam intensity, $\delta$ is retardance of the DIC prism, $\psi$ is angular orientation of its shear plane, $X$ is orientation of the principal plane of the polarization beam splitter, $\zeta$ is orientation of the fast axis of quarter-wave plate.

The measuring procedure of retardance derivative consists of the following steps. Before placing the DIC prism under investigation in the setup we look this prism between the crossed polarizers. The shear plane would be perpendicular to the observed color fringes or/ and the black fringe. Lets note the shear plane orientation.

Next we take out the quarter-wave plate from the setup, and put the DIC prism into the optical path. In this case, beam intensity on the screen is determined by the next expression:

$$
I^{\prime}=I \sin ^{2} \delta \sin ^{2} 2(\chi-\psi),
$$

By rotating the polarization beam splitter from $0^{\circ}$ to $180^{\circ}$ we achieve two extinctions of the laser spot on the screen. These extinctions differ by $90^{\circ}$. One of the extinction occurs when the beam splitter transmission axis is parallel to the shear plane of the DIC prism, which we noted. In this case the beam splitter angular scale $X$ points precisely the shear plane orientation $\psi$.

After that we place the quarter-wave plate QWP into the optical path. The fast axis of the quarter-wave plate has to makes an angle $45^{\circ}$ with the shear plane orientation, i.e. $\zeta=\psi$ $+45^{\circ}$. The beam splitter has to be also rotated by $45^{\circ}$. In this case $X_{0}=\psi+45^{\circ}$. Thus, the quarter-wave plate's fast axis is parallel to the beam splitter transmission axis. Laser spot on the screen becomes bright. Using formula (29) we could derive the following equation, which describes the spot's intensity: 


$$
I^{\prime}=I \sin ^{2}\left(\delta-2\left(\chi-\chi_{0}\right)\right)
$$

Rotating the beam splitter allows to extinguish the spot on the screen. The DIC prism retardance $\delta$ in a point of the laser beam incidence is determined by the simple formula:

$$
\delta=2\left(\chi-\chi_{0}\right), \quad(32)
$$

where $X$ is orientation angle of the beam splitter.

Using a linear stage we move the DIC prism in the horizontal direction and measure retardances $\delta_{1}$ and $\delta_{2}$ in two points $x_{1}$ and $x_{2}$. The retardance derivative along to the shear direction $X$ could be found by the following formula:

$$
\frac{d \delta}{d x}=\frac{\delta_{1}-\delta_{2}}{\left(x_{1}-x_{2}\right) \cos \psi}=\frac{2\left(\chi_{1}-\chi_{2}\right)}{\left(x_{1}-x_{2}\right) \cos \psi},
$$

Then we compute the shear angle (in radians):

$$
\varepsilon=\frac{\lambda}{180^{\circ}} \frac{\left(\chi_{1}-\chi_{2}\right)}{\left(x_{1}-x_{2}\right) \cos \psi} .
$$

Instead of finding retardance values in two points only, we can measure dependence of the analyzer angle $X$ on the laser beam position $x$ and use its derivative in formula above:

$$
\varepsilon=\frac{\lambda}{180^{\circ}} \frac{1}{\cos \psi} \frac{d \chi(x)}{d x} .
$$

In the described setup we utilized a generic green laser pointer with wavelength $532 \mathrm{~nm}$, beam splitting Glan-Thompson polarizer MSBTS-10-45 (Karl Lambrecht, Chicago, IL, http://www.klccgo.com), and precision achromatic quarter-wave plate AQ-100-0545 (Meadowlark, Frederick, CO, http://www.meadowlark.com). Because beam of our laser pointer has significant linearly polarized component we placed a generic circular polarizer for SLR camera between the pointer and the screen. In particularly we employed Tiffen circular polarizer 52HTCP (www.tiffen.com).

The measurement procedure could be simplified with using a custom built assembly with rotatable polarization beam splitter and quarter-wave plate [48]. This approach would not require taking out and putting in the quarter-wave plate.

Table 1 summaries some results of the shear angle measurements of various DIC prisms currently manufactured by Olympus (Tokyo, Japan, http://www.olympus.com). Highresolution DIC prism U-DICTHR has the smallest shear angle. The prism enables observations with high resolution but less glare even for thick specimen used in developmental and genetic research, such as finely structured diatoms, embryos, zebrafish and C. elegans. All-round prism U-DICT with the intermediate shear angle is suitable for observing a wide range of general specimens, such as tissue. High-contrast DIC prism UDICTHC has the largest shear angle. Using this prism the high contrast can be obtained even in high magnification observations of thin specimens, such as culture cells. 
According to our measurement the shear angle of the all-round DIC prism is double the high-resolution prism and half the high-contrast prism. Therefore some setups with the different objective lenses and the same condenser lens could employ the same condenser DIC prism. The following microscope configurations have interchangeable condenser DIC prisms:

- U-DICTHR prism \&10× objective, U-DICT prism \& 20× objective, U-DICTHC prism \& 40× objective;

- $\quad$ U-DICT prism \& 10× objective, U-DICTHC prism \& 20× objective;

- $\quad$ U-DICTHR prism \& 20× objective, U-DICT prism \& 40× objective;

- $\quad$ U-DICTHR prism \& 30× objective, U-DICT prism \& 60× objective;

- $\quad$ U-DICT prism \& 30× objective, U-DICTHC prism \& 60× objective.

For example, condenser DIC prism U-DIC40, which is designed for 40× objective lens and all around DIC prism U-DICT, could be also used with $20 \times$ lens and high-resolution DIC prism U-DICTHR. It is interchangeable with condenser prism U-DICT20HR. Condenser prisms U-DIC20 and U-DIC40HC could be also mutually replaced.

We also found that focal distance of the dry top condenser lens is double the oil top condenser lens. Hence, the condenser prism designed for the oil condenser would work the dry condenser and the one step lower resolution objective prism. From another hand, the condenser prism designed for the dry condenser could be paired the oil condenser and the one step higher resolution objective prism. For instance, the dry condenser DIC prism UDIC60HC, which is designed to work with $60 \times$ objective lens and high-contrast prism UDICTHC, would also work with oil condenser and all around prism U-DICT. So, prism UDIC60HC is interchangeable with prism U-ODICT60. Then it is also could work instead of U-ODIC30HR, as mentioned early. Another example of interchangeable pair of prisms is UDIC100 and U-ODIC100HR. The first prism is designed for dry top condenser lens, 100x oil objective and U-DICT prism. The second prism is intended for oil top condenser lens, $100 \times$ oil objective and U-DICTHR prism.

The Table 1 shows the corresponding computed shear distance $d$ in the object plane and ratio of the shear distance to the Airy disk radius $d / r_{\text {Airy }}$. For the calculation we used standardized reference focal lengths of the tube lenses $L_{t}$ for infinity-focused objective lens, which are adopted by several microscope manufacturers.

$$
d=\varepsilon \frac{L_{t}}{M}
$$

where $M$ is objective lens magnification. In particularly, the reference focal length $L_{t}$ is 180 $\mathrm{mm}$ for Olympus, $164.5 \mathrm{~mm}$ for Zeiss, and $200 \mathrm{~mm}$ for Nikon and Leica microscopes [6].

Radius of Airy disk $r_{\text {Airy }}$ is determined by the following equation [6]:

$$
r_{\text {Airy }}=0.61 \frac{\lambda}{N A},
$$

where $N A$ is objective lens numerical aperture.

The above data is shown for the green light $(\lambda=532 \mathrm{~nm})$. The shear angle was about $4 \%$ less for the red light $(\lambda=641 \mathrm{~nm})$. 
We have measured parameters of Olympus DIC prism U-DICTH, which was manufactured in previous years. Its shear angle is the same as the prism U-DICTHR. Mehta and Sheppard [44] found the angular shear $74 \mu \mathrm{rad}$ for U-DICTS prism at wavelength $550 \mathrm{~nm}$. This shear corresponds exactly to our result for U-DICT prism. Other our results indicate that shear angles of Nikon 60×I and Zeiss PA63×/1.40III DIC sliders are $76 \mu \mathrm{rad}$ and $71 \mu \mathrm{rad}$, correspondently. According to formula (36) the Nikon slider and $60 \times$ objective lens create the shear distance $0.25 \mu \mathrm{m}$, and the Zeiss slider and $63 \times$ objective lens introduce shear $0.18 \mu \mathrm{m}$.

\section{Appendix B: Calibration of LC variable retarder for bias modulation}

Another parameter, which has to be quantified, is a bias amount introduced by the phase shifter. Any method for measuring retardance could be used to find a dependence of bias on the applied voltage. For example, one could employ the return-path compensation method, which we described in Appendix A. However all calibration methods would require taking the phase shifter out of a microscope and placing it into a separate measuring setup. But on the other hand a phase shift of LC variable retarder depends on the temperature, wavelength, set of incidence angles of the passing rays, etc. Therefore it would be convenient to calibrate the LC variable retarder directly in the OI-DIC microscope (Fig. 1 and Fig. 2) in order to avoid mismatch between the calibration and actual data.

For measuring dependence of the bias on the applied voltage we could use preliminary calibrated DIC prims. In a transmitted DIC microscope the total optical path difference (bias) between two interfering beams is created by a pair of DIC prisms. One of them is located in the imaging part, and another in the illumination part. Each of the prisms introduces own bias, which is not uniform and has a gradient. However, the prisms are placed and oriented in that way that they mutually compensate the bias gradient. Thus, the total bias distribution becomes even across the objective back focal plane. The bias can be changed by a lateral shifting of one of the DIC prisms along the bias gradient direction. As it was shown in Appendix A (formula (26)), the unitless bias gradient equals to shear angle (in radians). We can write linear equation for the current bias $\Lambda(x)$ :

$$
\Lambda(x)=\tilde{\Lambda}+\varepsilon\left(x-x_{0}\right),
$$

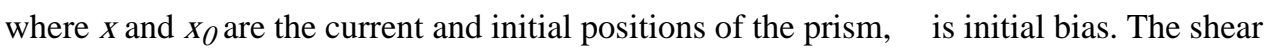
angle $\epsilon$ can be found in Table 1 or it can be measured, as it described in Appendix A.

Usually DIC prism is shifted by a translation screw. Then the bias variation could be determined using the pitch of a screw thread $p$ and revolution number $R$ in the following way:

$$
\Lambda(R)=\tilde{\Lambda}+\varepsilon p R .
$$

For example, Olympus high-resolution DIC prism U-DICTHR has translation screw with pitch $2.5 \mathrm{~mm}$. The screw allows maximum 5 rotations. According to the Table 1 the prism shear angle is $40 \mu \mathrm{rad}$. Using equation (15) we receive the bias variation $\Lambda=100 \mathrm{~nm}$ per screw $360^{\circ}$-turn and the total range of the bias change $\Lambda_{\text {tot }}=500 \mathrm{~nm}$. General Olympus DIC prism U-DICT has $3 \mathrm{~mm}$ screw pitch and maximum 5 rotations. Consequently its bias variation $\Lambda$ is $225 \mathrm{~nm}$ per screw turn and the total bias range $\Lambda_{\text {tot }}$ is $1125 \mathrm{~nm}$.

Let's consider OI-DIC microscope for the transmitted light (Fig.1), for instance. Before calibration the phase shifter and polarization rotators are removed from the microscope. An 
empty area of the specimen under investigation is placed in the center of view field. The DIC prisms are adjusted for the maximal image darkness. That means the prisms introduce zero total bias. Then the phase shifter is put into the place.

Because the polarizer and analyzer are crossed and their transmissial axes are oriented at $45^{\circ}$ to the principal axes of the orthogonal DIC prisms the beam intensity in the image central area $I\left(V_{r m s}\right)$ could be described by the following equation:

$$
I\left(V_{r m s}\right)=\tilde{I} \sin ^{2}\left(\frac{\pi}{\lambda}\left(\Lambda_{\sum}+\Gamma\left(V_{r m s}\right)\right)\right)+I_{c}
$$

where $\tilde{I}$ is beam intensity after the polarizer, $\Lambda_{1}, \Lambda_{2}, \Lambda_{3}, \Lambda_{4}$ and are biases contributed by prisms DIC1, DIC2, DIC3, and DIC4, respectively, $\Lambda_{\Sigma}=\Lambda_{1}-\Lambda_{2}-\Lambda_{3}+\Lambda_{4}$ is combined bias introduced by all prisms, $\Gamma\left(V_{r m s}\right)$ is bias created by the phase shifter, $V_{r m s}$ is effective (rms) value of the applied voltage, and $I_{\mathcal{C}}$ corresponds to a constant offset of the intensity signal.

If the setup is adjusted for the maximal extinction then biases $\Lambda_{1}, \Lambda_{2}, \Lambda_{3}$, and $\Lambda_{4}$ compensate each other and $\Lambda_{\Sigma}=0$. Thus, in the initial state of the OI-DIC setup, a dependence of beam intensity $I_{O}\left(V_{r m s}\right)$ on the applied voltage is determined by the formula:

$$
I_{0}\left(V_{r m s}\right)=\tilde{I}_{\sin ^{2}}\left(\frac{\pi}{\lambda} \Gamma\left(V_{r m s}\right)\right)+I_{c}
$$

Next we move laterally one of the DIC prisms by the same distance by rotating the translation screw in the clockwise and counterclockwise directions. According to the linear equation (15) in these cases the prism bias will change by amount $\pm(\in p R)$. The correspondent dependences of the beam intensity $I_{-1}\left(V_{r m s}\right)$ and $I_{1}\left(V_{r m s}\right)$ are determined by the following equations:

$$
\begin{gathered}
I_{-1}\left(V_{r m s}\right)=\tilde{I}_{\sin ^{2}}\left(\frac{\pi}{\lambda}\left(\Gamma\left(V_{r m s}\right)-\varepsilon p R\right)\right)+I_{c} \\
I_{1}\left(V_{r m s}\right)=\tilde{I} \sin ^{2}\left(\frac{\pi}{\lambda}\left(\Gamma\left(V_{r m s}\right)+\varepsilon p R\right)\right)+I_{c}
\end{gathered}
$$

Using the measured beam intensities $I_{O}\left(V_{\text {Ims }}\right), I_{-1}\left(V_{\text {rms }}\right)$ and $I_{1}\left(V_{\text {rms }}\right)$ we could obtain the phase shifter bias $\Gamma\left(V_{\text {rms }}\right)$ :

$$
\Gamma\left(V_{r m s}\right)=\frac{\lambda}{2 \pi} \arctan \left[\frac{I_{1}\left(V_{r m s}\right)-I_{-1}\left(V_{r m s}\right)}{I_{1}\left(V_{r m s}\right)+I_{-1}\left(V_{r m s}\right)-2 I_{0}\left(V_{r m s}\right)} \tan \left(\frac{\pi}{\lambda} \varepsilon p R\right)\right] .
$$

The bias computation formula (44) yields values within $[0, \lambda]$ interval. In other words, without further analysis, the bias measurement cannot distinguish a certain bias value, say $\Gamma_{0}, \Gamma_{0}+\lambda$. It is said that the measurement yields wrapped bias information. However, using prior knowledge regarding the continuity of the bias variation, the obtained result can be numerically corrected to cover greater intervals, outside $[0, \lambda]$, by mathematical operation called phase unwrapping. Mathematically, the unwrapping operation essentially searches for $2 \pi$ jumps in the signal and corrects them by adding the $2 \pi$ values back to the signal. For example, Matlab ${ }^{\mathrm{TM}}$ has very efficient and fast standard command called "unwrap". 
In order to build the correct bias dependence it is also necessary to take into account a type of LC cell, which is employed in the phase shifter. In particular, retardance of conventional Freedericksz ECB cell decreases with applied voltage. The retardance is minimal and equals about $0.1 \lambda$ at the highest voltage. In a case of VA ECB cell the retardance is minimal and equals zero when no voltage is applied.

Similar bias calibration method would work for the reflected light OI-DIC setup (Fig. 2). Because each DIC prism is used twice, in the illumination and reflected beams, the bias introduced by lateral movement of the prism is doubled. Therefore formula (44) for the reflected light OI-DIC has to be modified in the following way:

$$
\Gamma\left(V_{r m s}\right)=\frac{\lambda}{2 \pi} \arctan \left[\frac{I_{1}\left(V_{r m s}\right)-I_{-1}\left(V_{r m s}\right)}{I_{1}\left(V_{r m s}\right)+I_{-1}\left(V_{r m s}\right)-2 I_{0}\left(V_{r m s}\right)} \tan \left(\frac{2 \pi}{\lambda} \varepsilon p R\right)\right] .
$$




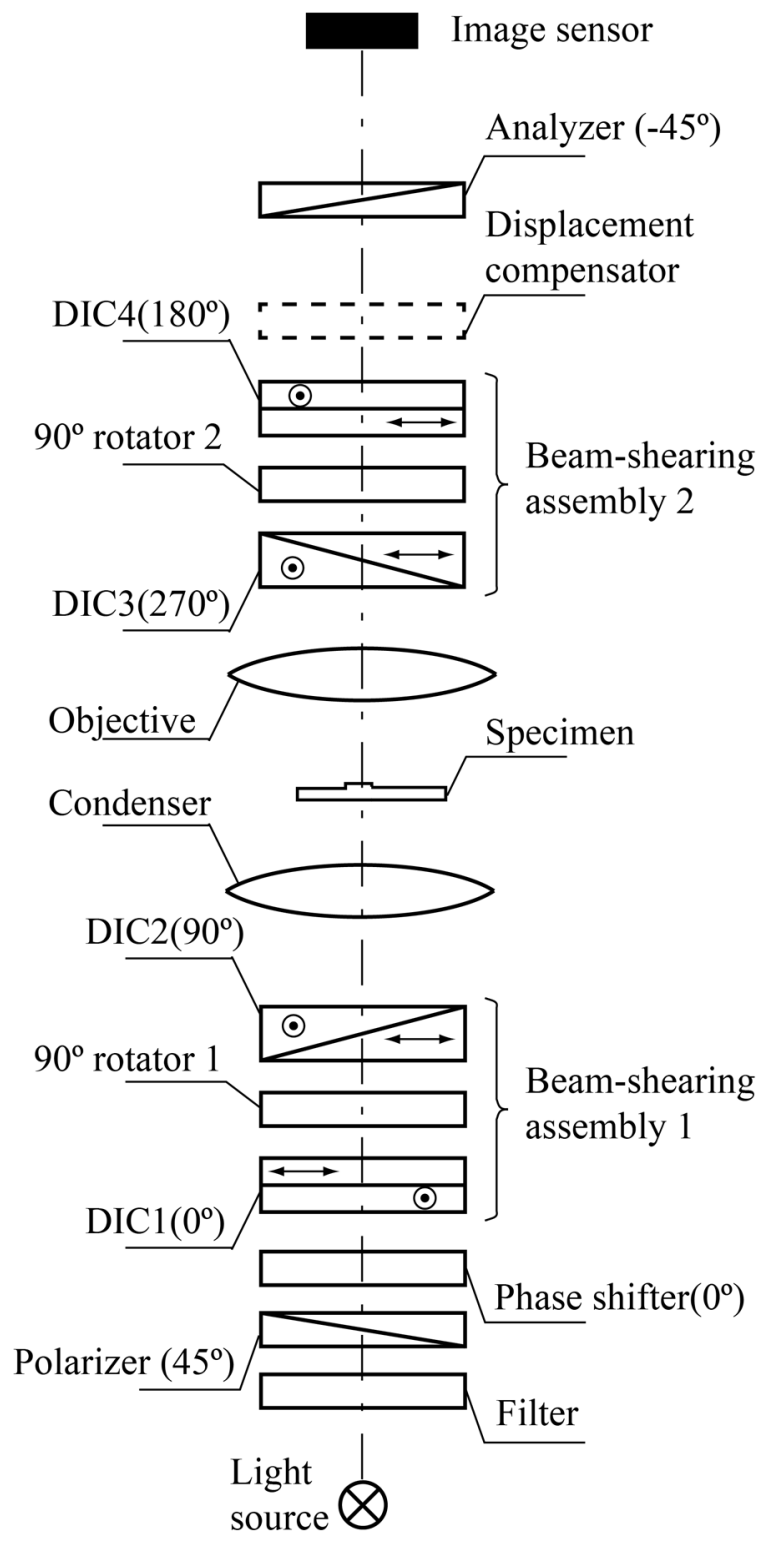

Fig. 1.

Principal optical schematics of OI-DIC microscope for the transmitted light. 


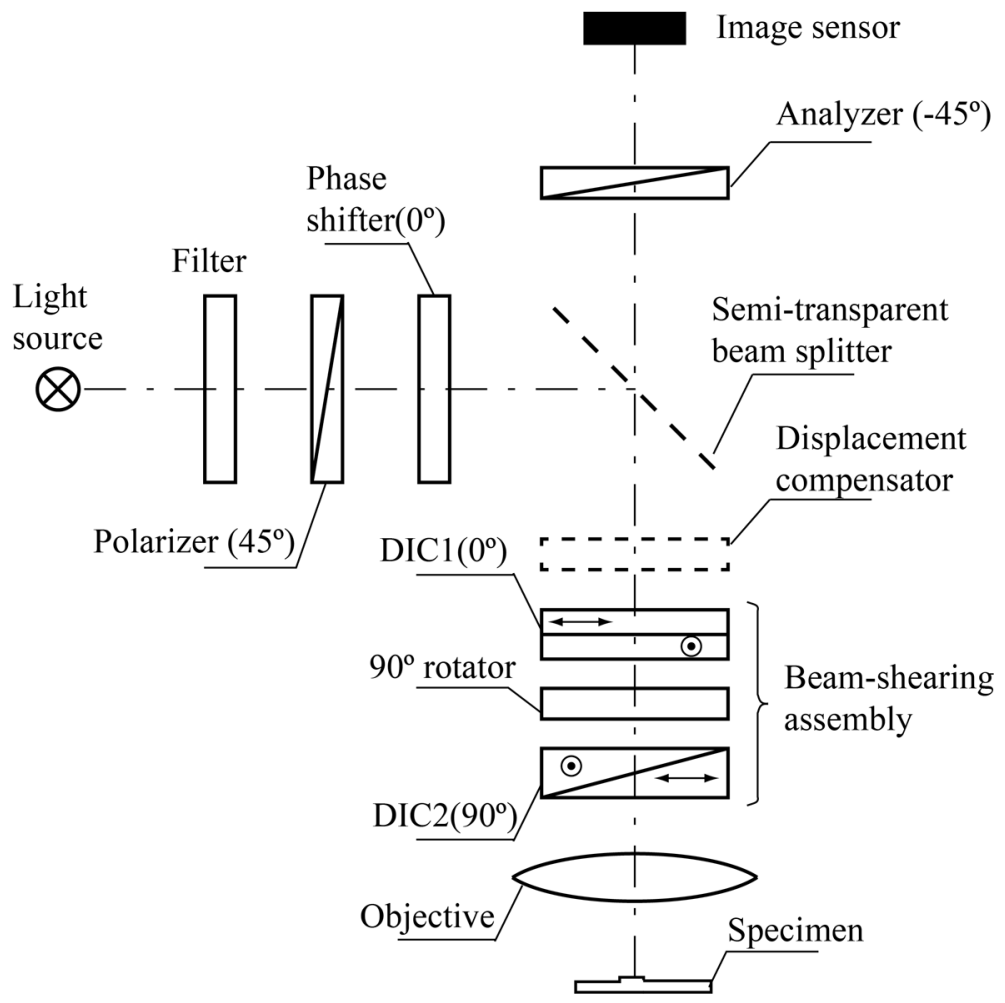

Fig. 2.

Principal optical schematics of OI-DIC microscope for the reflected light. 


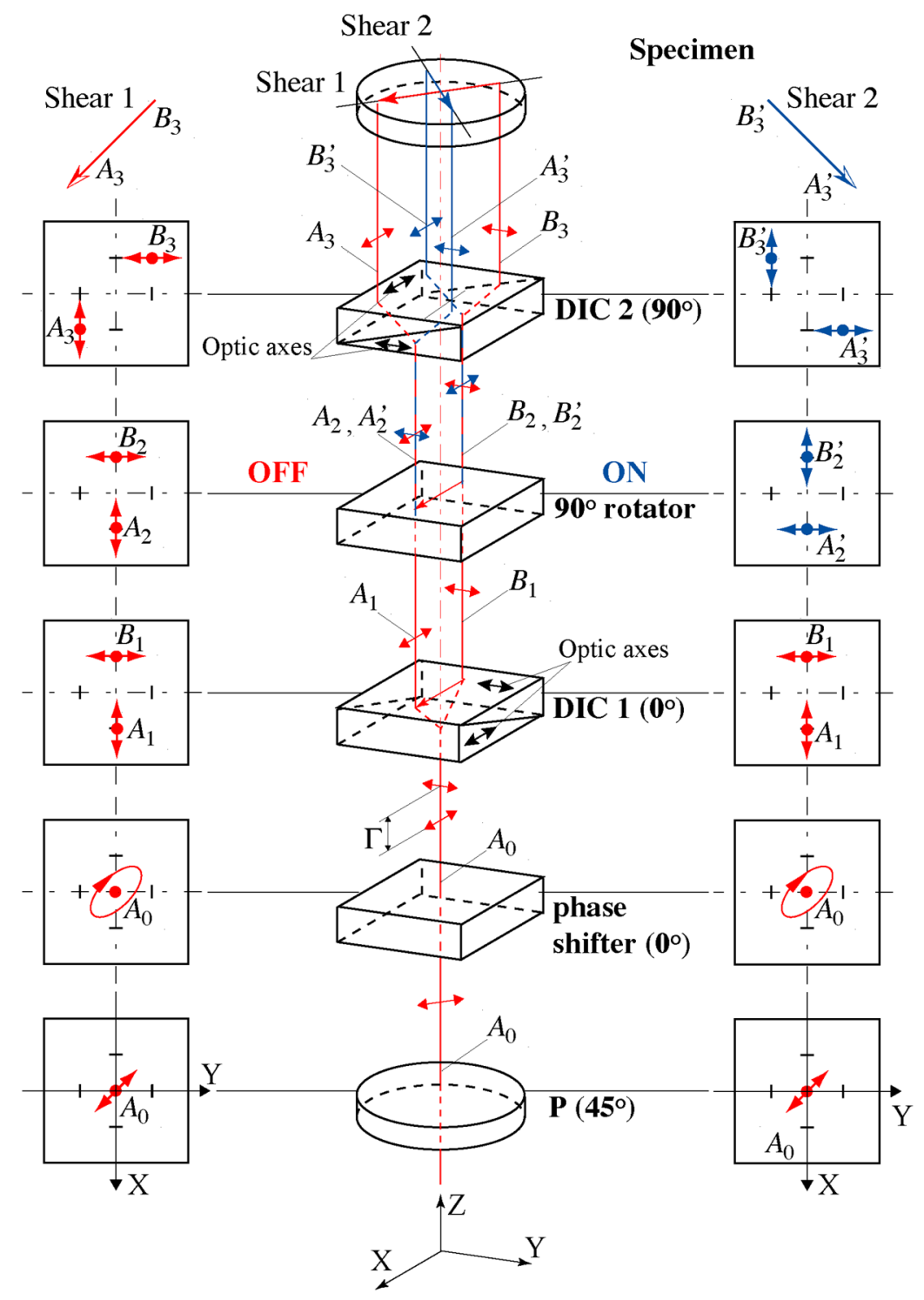

Fig. 3.

Design and principle of work of beam-shearing assembly (Color online). 


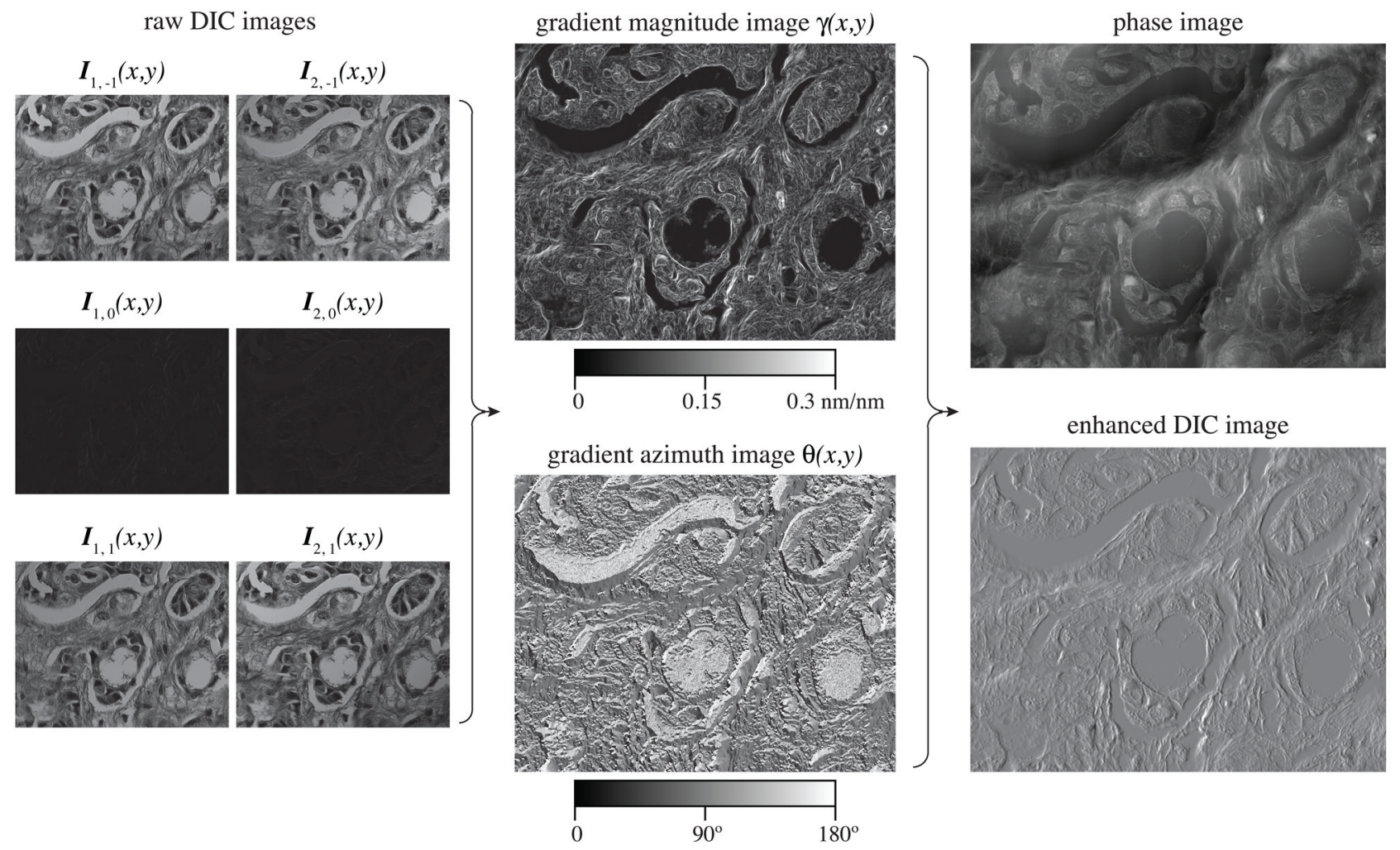

Fig. 4.

OI-DIC images of the hematoxylin-eosin (H\&E) stained breast cancer tissue sample. 


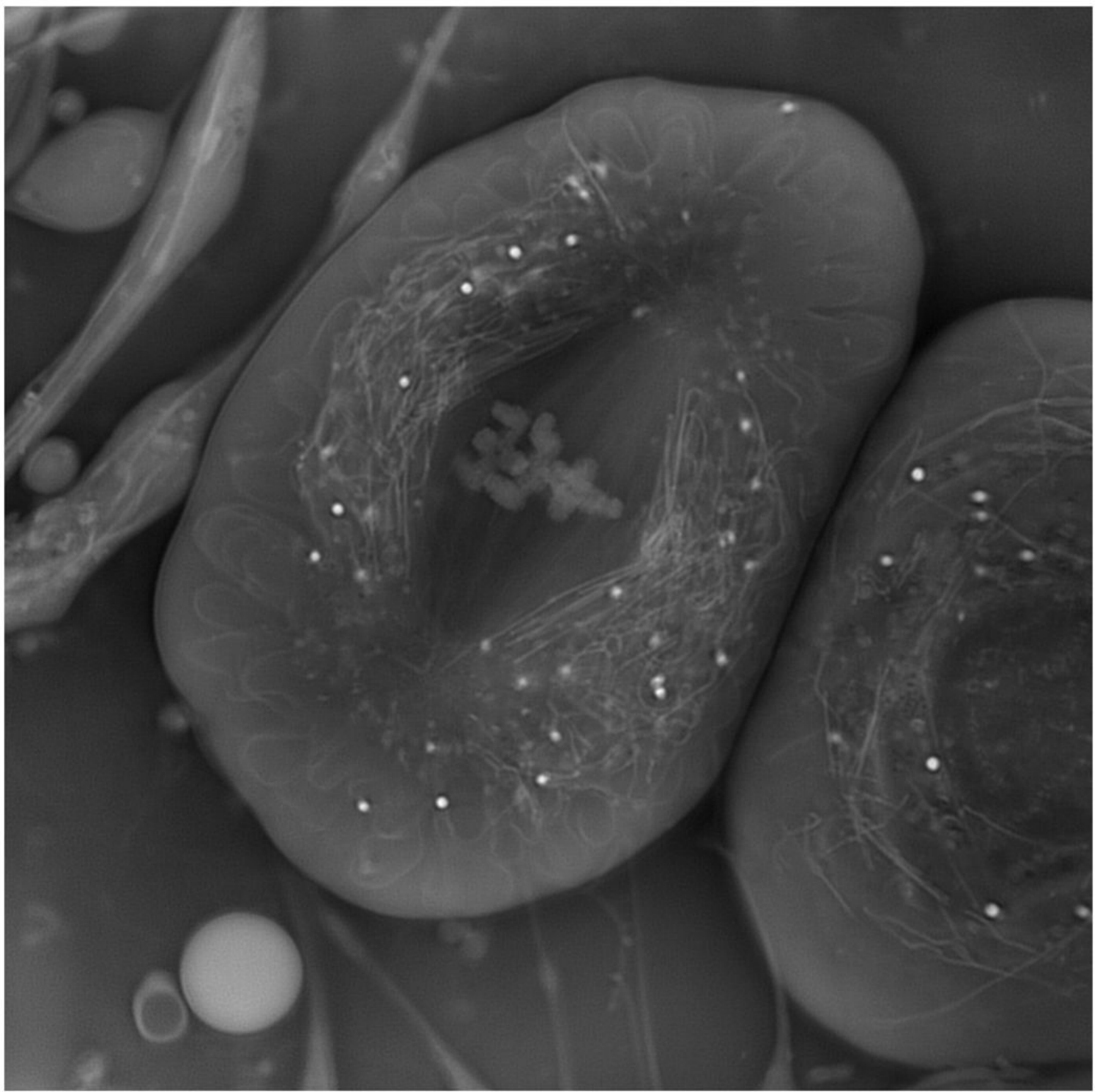

Fig. 5.

Crane fly spermatocyte (full metaphase of meiosis-I). OI-DIC phase image; brightness is linearly proportional to refractive index. 

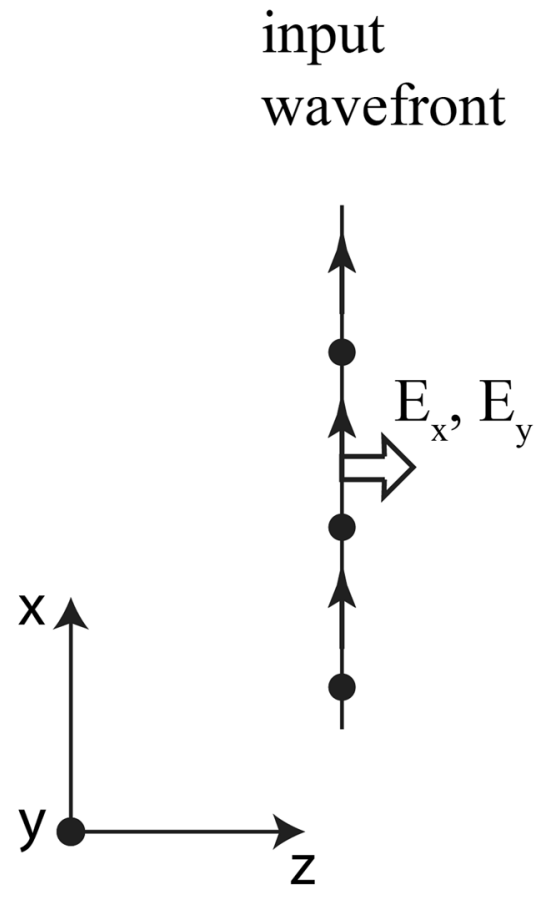
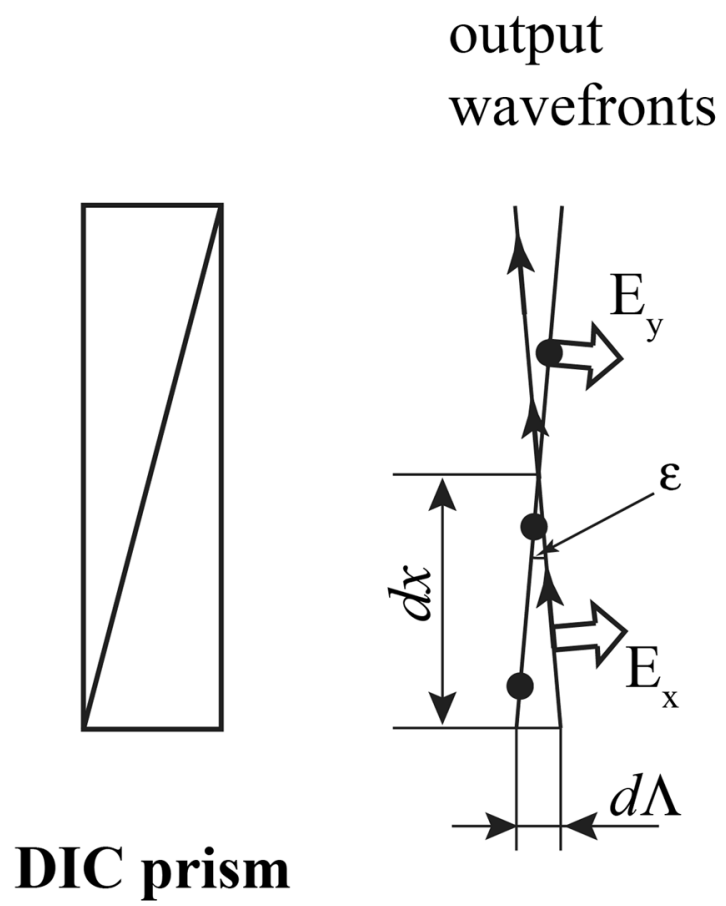

Fig. 6.

Angular splitting the incident beam with two orthogonal polarization components $E_{x}$ and $E_{y}$ by DIC prism into two output beams with shear angle $\epsilon$. 


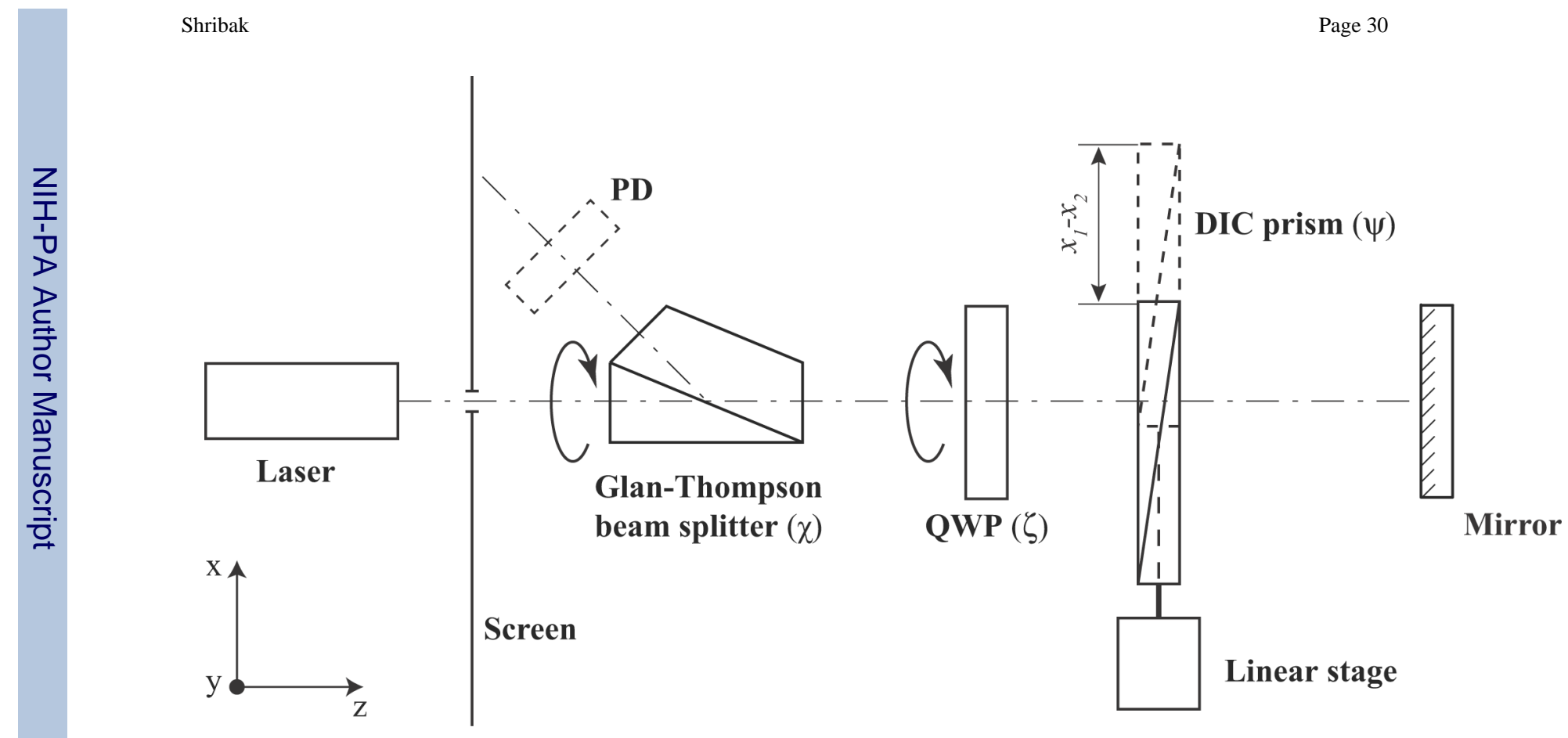

Fig. 7.

Setup for measuring shear angle of DIC prism with employing the return-path birefringence compensator. 


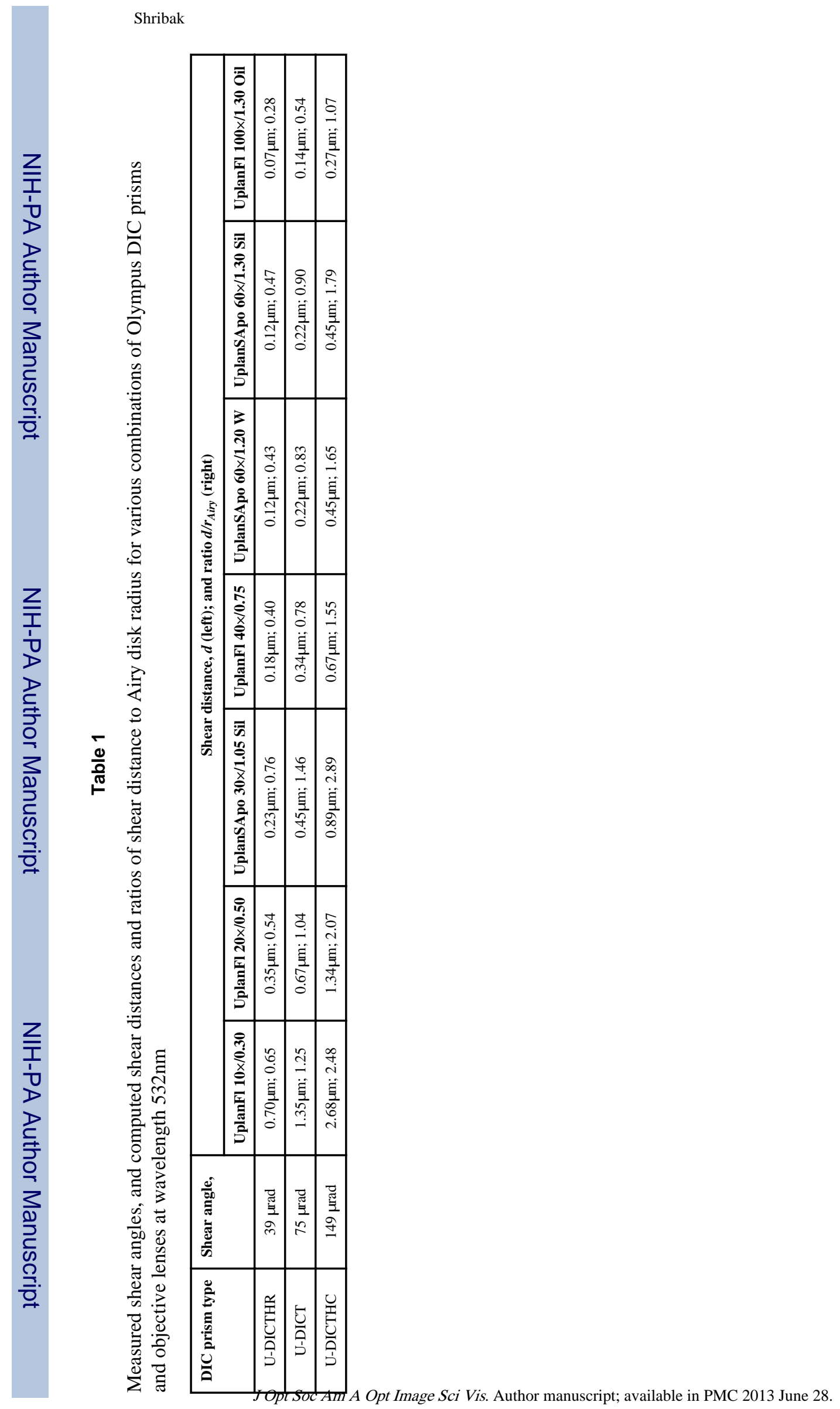

Page 31 